Geert Keil

\title{
WAS LEHRT UNS DAS GETTIER-PROBLEM ÜBER DAS VERHÄLTNIS ZWISCHEN INTUITIONEN UND BEGRIFFSANALYSEN?
}

\section{ZUSAMMENFASSUNG}

Der Beitrag beleuchtet einen bisher kaum gewürdigten Grund dafür, dass die Gettier-Debatte nicht zu einer systematisch verbesserten Analyse des Wissensbegriffs geführt hat. Es wird die These entwickelt und verteidigt, dass diejenigen Komplikationen, die einen Gettierfall zu einem solchen machen, sich stets in den blinden Flecken der Situationsrepräsentation des epistemischen Subjekts befinden. Diese These ist in die metaphilosophische Fragestellung eingebettet, was das Gettierproblem uns über das Verhältnis von sprachlichen Intuitionen und Begriffsanalysen lehrt. Es gibt unter kompetenten Sprechern beträchtliche Einmütigkeit darüber, dass paradigmatische Gettierfälle als Fälle von Nichtwissen zu klassifizieren sind, aber ungleich weniger Einigkeit darüber, ob und wie die klassische Analyse des Wissensbegriffs verbessert werden kann. $\mathrm{Zu}$ der Frage, warum es so schwer ist, konvergierende sprachliche Intuitionen über Einzelfälle in eine gettierfallsichere allgemeine Analyse zu überführen, werden folgende Thesen entwickelt: Was in Gettierfällen konfligiert, sind nicht Analyse und Intuition als solche, sondern die Charakterisierung eines Situationstyps und die Beurteilung einer einzelnen Situation angesichts einer bestimmten Gettierkomplikation. Die Aufgabe, eine allgemeine Beschreibung der mit Wissen unvereinbaren Komplikationen zu geben, geht weit über die kompetente Beurteilung von Einzelfällen hinaus. Möglicherweise ist sie unlösbar, weil das wörtliche Zutreffen der Beschreibung einer epistemischen Situation niemals garantieren kann, dass sich in den Leerstellen der Beschreibung keine Gettierkomplikation verbirgt.

\section{EINLEITUNG}

Seit Edmund Gettier 1963 auf eine Unzulänglichkeit der klassischen dreiteiligen Analyse des Wissensbegriffs aufmerksam machte, ist auf das sogenannte Gettierproblem eine Unmenge Tinte verwendet worden. Wer ihr nur 
den kleinsten Tropfen hinzufügen möchte, ist gut beraten, sich Rechenschaft über sein genaues Erklärungs- oder Klärungsziel abzulegen.

Den Hauptstrang der Gettierdebatte bilden Versuche, Gettiers Herausforderung für die klassische Wissensanalyse durch eine Verbesserung dieser Analyse zu begegnen. Die Herausforderung wurde und wird darin gesehen, dass die von Gettier präsentierten Beispielfälle die drei Bedingungen, nach denen Wissen gerechtfertigte wahre Überzeugung ist, erfüllen oder zu erfüllen scheinen, ohne dass kompetente Sprecher diese Fälle intuitiv als Fälle von Wissen klassifizieren würden. Die klassische Analyse scheint keine hinreichende Bedingung für Wissen anzugeben. ${ }^{1}$

Die Herausforderung bestand also darin, die beste verfügbare Analyse eines philosophischen Grundbegriffs und die sprachlichen Intuitionen hinsichtlich der Beurteilung einzelner Fälle, die unter diesen Begriff fallen, wieder zur Deckung zu bringen. Abhilfe wurde darin gesehen, die Analyse so abzuwandeln, dass sie Gettierfälle aus dem Wissensbegriff ausschließt - entweder durch Hinzufügung einer vierten Bedingung oder durch eine Präzisierung der Bedingung des Gerechtfertigtseins. Diese Verbesserung wurde und wird in der Regel als eine Verfeinerung der klassischen Wissensanalyse verstanden, da die große Mehrheit der Erkenntnistheoretiker der Auffassung ist, dass die klassische Analyse immerhin notwendige Bedingungen für Wissen angibt und, von den Gettierfällen abgesehen, die Extension des Wissensbegriffs recht gut trifft. Nur wenige Theoretiker plädieren dafür, eine der drei Bedingungen überhaupt aufzugeben. Gettierfälle erscheinen insofern als ein Spezialproblem am Rande der Extension des Wissensbegriffs, das im Prinzip beherrschbar sein sollte.

Bis heute liegt indes keine konsensfähige Verbesserung der klassischen Wissensanalyse vor. Vielmehr ist in der Debatte eine gewisse Ermüdung eingetreten. Man spricht seit geraumer Zeit despektierlich von einer GettierIndustrie; viele Debattenteilnehmer haben den Eindruck, es sei schon alles ausprobiert worden und es kämen allenfalls noch Verfeinerungen bestehender Vorschläge hinzu. Dieser Eindruck ist nicht ganz richtig, denn die Gettierdebatte hat durch die Entwicklung differenzierter Antizufallstheorien und insbesondere durch die intensive Diskussion von safety accounts seit den 90er-Jahren beträchtliche Fortschritte gemacht. Gleichwohl herrschen mittlerweile Beiträge vor, die nicht mehr nach einer Verbesserung der klassischen Wissensanalyse streben, sondern nach einer Diagnose der Fruchtlosig-

\footnotetext{
1 Wegen der Prozess/Produkt-Ambiguität von „Begriffsanalyse“ ist der Hinweis am Platze, dass im Titel dieses Beitrags und in der Rede von der „klassischen Analyse des Wissensbegriffs“ die Produkt-Lesart aktualisiert ist. In späteren Abschnitten steht die Prozess-Lesart im Fokus. Intuitionen mit dem begriffsanalytischen Verfahren zu kontrastieren wäre irreführend, weil Intuitionen in der „method of cases“" selbst eine zentrale Rolle in diesem Verfahren spielen.
} 
keit dieser Versuche. Williamson hat die Suche nach einer reduktiv analytischen Wissensdefinition, in die das Gettierproblem eingebettet ist, als ein degenerierendes Forschungsprogramm bezeichnet (vgl. Williamson 2000, 31). Verschiedene Philosophen sind einen Schritt zurückgetreten und haben die Gettierdebatte als ganze zum Anlass eines meta-wissensanalytischen oder sogar metaphilosophischen Kommentars genommen. ${ }^{2} \mathrm{Zu}$ diesen Reaktionen gehören insbesondere:

(a) Die klassische Wissensanalyse ist einem überholten Verständnis von Begriffsanalyse verpflichtet, nämlich dem Projekt der Angabe einer Menge notwendiger und gemeinsam hinreichender Bedingungen für die Anwendbarkeit eines Begriffs. Beispiele erfolgreicher reduktiver Begriffsanalysen sind in der Erkenntnistheorie wie außerhalb schwer zu finden. Wenn aber die Struktur unseres Begriffssystems nicht so ist, dass es Analysen der genannten Art zulässt, beweist das Scheitern der Versuche, die Analyse des Wissensbegriffs gegen Gettierfälle immun zu machen, nichts erkenntnistheoretisch Interessantes. $^{3}$

(b) Wissenszuschreibungen sind kontextabhängig. Eine befriedigende generelle Analyse des Wissensbegriffs quer zu allen Kontexten wird sich nicht finden lassen, weil insbesondere die für Wissen erforderlichen Rechtfertigungsstandards mit dem epistemischen Kontext des Wissenszuschreibers variieren. Allein die Einsicht in die kontextuelle Variabilität von Rechtfertigungsstandards - das Hauptmotiv für den erkenntnistheoretischen Kontextualismus - kommt allerdings noch keiner Abkehr von der klassischen Wissensanalyse gleich, solange die Rechtfertigungsbedingung, wie auch immer sie kontextuell oder sogar indexikalisch spezifiziert wird, gemeinsam mit den beiden anderen Komponenten eine notwendige Bedingung für Wissen bleibt. Eine das Gettierproblem direkt adressierende Variante des Kontextualismus behauptet, dass es sich beim Gettierproblem um ein Pseudoproblem handle, das auf einer unzulässigen Vermischung der Perspektiven und Standards des Subjekts, das sich selbst zu Recht Wissen zuschreibt, und des externen Beobachters, der ihm Wissen abschreibt, beruht (vgl. Tolksdorf 2013).

(c) Von Seiten der experimentellen Philosophie ist das Gettierproblem als ein Artefakt von Erkenntnistheoretikern kritisiert worden, die ihre eigenen Expertenintuitionen auf den Wissensbegriff projizieren. Tatsächlich variierten die sprachlichen Intuitionen angesichts von Gettierfällen mit der ethni-

\footnotetext{
${ }^{2}$ Vgl. auch Berneckers (2013) hilfreiche Unterscheidung zwischen „Lösungsversuchen“ und „Auflösungsversuchen" des Gettierproblems.

${ }^{3}$ Vgl. zum Beispiel Baumann (2013). Williamson zufolge ist Wissen ein mentaler Zustand, der sich nicht in Komponenten zerlegen lässt. Der Wissensbegriff sei nichtanalysierbar und gegenüber den Begriffen des Überzeugtseins und des Gerechtfertigtseins basal (,knowledge first“, vgl. Williamson 2000).
} 
schen und mit der Geschlechtszugehörigkeit der befragten Personen (Weinberg et al. 2001, Buckwalter und Stich 2011). Sorgfältige Nachfolgestudien von Nagel et al. (2013) haben diese Befunde indes nicht bestätigt: GettierIntuitionen ${ }^{4}$ sind über verschiedene Menschengruppen hinweg recht stabil.

(d) Der klassisch analysierte Wissensbegriff ist ein Hybridbegriff, der sich aus kategorial heterogenen Komponenten zusammensetzt: „knowing is a hybrid of warranted belief, which is mental, and truth, which is not" (Quine 2000, 415). Ausgehend von dieser Diagnose haben Quine, Sartwell und Beckermann vorgeschlagen, den klassisch analysierten Wissensbegriff in der Erkenntnistheorie überhaupt zu den Akten zu legen und statt dessen mit seinen Komponenten weiterzuarbeiten.

Diese Reaktionen haben jeweils einen berechtigten Kern, doch ist keine von ihnen geeignet, die Herausforderung des Gettierproblems als gegenstandslos, als überholt oder als Scheinproblem zu erweisen. Auch metaphilosophisch motivierte Abgesänge auf das Projekt der Analyse des Wissensbegriffs erscheinen verfrüht. Fünfzig Jahre sind für die Klärung eines schwierigen begrifflichen Problems keine lange Zeit. Zwischen dem Theaitet und Gettiers Aufsatz hat man mehr als 2.300 Jahre lang auf einen substanziellen Fortschritt warten müssen. ${ }^{5}$

Auch mein Beitrag kreist um eine metaphilosophische Frage, nämlich um die, was Gettierfälle uns über das Verhältnis von Begriffsanalysen und sprachlichen Intuitionen lehren. Eine Antwort auf diese Frage wird weder die klassische Wissensanalyse verbessern noch das Gettierproblem als Scheinproblem entlarven. Sie kann aber dazu beitragen, besser zu verstehen, worin die Herausforderung der Gettierfälle besteht und warum ihr so schwer zu begegnen ist.

\section{EINE FubNOTE ZU Platon: DAS BEISPIEL DER PERSONENERKENNUNG}

Die klassische dreiteilige Analyse des Wissensbegriffs wird in der Literatur häufig als Platons Analyse bezeichnet, weil sie im Dialog Menon verteidigt

\footnotetext{
${ }^{4}$ Mit dem Ausdruck „Gettier-Intuition“ bezeichne ich eine Intuition mit dem Inhalt „Dieser (aktuelle oder hypothetische) Fall ist ein Fall einer wahren, gerechtfertigten Überzeugung, die kein Wissen ist".

${ }^{5}$ Vielleicht auch etwas weniger lange: Gettierfälle sind schon in der indo-tibetischen Philosophie des 8. Jahrhunderts (vgl. Stoltz 2007) sowie im späten 14. Jahrhundert von Peter von Mantua diskutiert worden (vgl. Martens 2011). Die tibetischen Philosophen scheinen Gettierfälle entdeckt zu haben, nicht aber das Gettierproblem, denn sie diskutierten die Fälle als Beispiele dafür, dass weder Rechtfertigung noch Wissen vorliegt (vgl. Stoltz 2007, 398-9). - Den Hinweis auf diese historischen Debatten verdanke ich Peter Baumann und Sven Bernecker.
} 
und im (späteren) Theaitet ausführlich entwickelt und diskutiert wird. Die letzte der von Sokrates und seinem Gesprächspartner erwogenen Antworten auf die Frage, was epistêmê sei, lautet „meta logou alêthês doxa“, also mit Erklärung verbundene wahre Meinung (Theaitet 201c, 202 b-c). Nun hält Sokrates diese letzte Antwort zwar für besser als die zuvor erwogenen, ironisiert sie aber umgehend und hält sie offenkundig für unzulänglich: „Also hätten wir auf diese Weise am heutigen Tage erreicht, was seit langer Zeit viele Weisen gesucht und, ohne es zu finden, alt geworden sind?" (202d). Sokrates' Bedenken betreffen die Logos-Bedingung, die ihm präzisierungsbedürftig erscheint. Er diskutiert drei mögliche Interpretationen von „logos“, deren letzte er am Beispiel der Personenerkennung erläutert:

Wenn du das Unterscheidende eines Dinges auffaßt, wodurch es von den übrigen verschieden ist, so behaupten einige, du habest seine Erklärung aufgefaßt. Solange du aber noch etwas Gemeinschaftliches triffst, so würde deine Erklärung auf dasjenige gehen, was zu dieser Gemeinschaftlichkeit gehört. (208d)

Um eine bestimmte Person zu identifizieren, genüge es zum Beispiel nicht, sie mithilfe eines Merkmals wie „Krummnasigkeit“" $\mathrm{zu}$ charakterisieren. Viele Menschen haben krumme Nasen; gesucht ist etwas, ,wodurch sich das Gefragte von allen übrigen Dingen unterscheide“ (208c):

Nicht eher, glaube ich, wird Theaitetos in mir vorgestellt werden, bis diese Krummnasigkeit selbst ein sie von andern Krummnasigkeiten, die ich auch schon gesehen, unterscheidendes Merkmal in mir abdrückt [...]. (209c)

Erst ein solches distinktives Merkmal (sêmeion) gewährleistet die erforderliche Qualität der Erklärung. Eine Übertragung der Distinktivitätsbedingung von der nominalen Einstellung des Vorstellens oder (Wieder-)Erkennens auf die propositionale Einstellung des Wissens, dass p, ist nicht trivial. Ein Versuch: Um aus wahrer Meinung Wissen zu machen, muss die fragliche Rechtfertigung oder Erklärung spezifisch sein. Sie muss eine maximal kontrastive Erklärung sein, die die fragliche Meinung erklärt und keine davon leicht abweichende.

Die Forderung nach Distinktivität oder Spezifität lässt sich als Kommentar zum Gettierproblem avant la lettre auffassen. Platon hat im Schlussteil des Theaitet keine Gettierfälle präsentiert, aber zumindest einen Zipfel des Gettierproblems erhascht. Er hat insbesondere erkannt,

- dass die dreigliedrige Analyse noch nicht perfekt ist,

- dass die problematischste Komponente die Logos-Bedingung ist,

- dass diese Bedingung der Präzisierung bedarf, um nicht zu liberal zu sein, 
- dass die Präzisierung auf große Probleme stößt. ${ }^{6}$

Überdies wäre Platons Präzisierungsidee, wenn man sie ausarbeitete, vermutlich geeignet, viele Gettierfälle aus dem Wissensbegriff auszuschließen. Am Beispiel des ersten der beiden Originalbeispiele: Eine Charakterisierung eines Stellenbewerbers, die auf alle Personen zutrifft, die zehn Münzen in der Tasche haben, ist eben nicht distinktiv. Hätte Smith seinen Mitbewerber mithilfe einer identifizierenden Beschreibung - einer definite description - gekennzeichnet, so wäre sein Irrtum offen zutage getreten. Die geäußerte Überzeugung wäre falsch gewesen, mithin kein Gettierfall.

Leider teilt Platons Präzisierungsidee einen gravierenden Nachteil mit etlichen Lösungsvorschlägen für das Gettierproblem, etwa mit der No false lemmas-Bedingung und mit einigen Antizufallstheorien. Durch die Zusatzauflage werden die Standards für eine Rechtfertigung und damit für Wissen so anspruchsvoll, dass sie auch in Fällen, die für Nichtskeptiker klare Fälle von Wissen sind, nicht mehr erfüllt werden. Personenerkennung ist dafür ein gutes Beispiel. Wann kann man davon sprechen, dass man eine Person wiedererkannt hat? Die Polizei spricht von der Identifizierung eines Täters, wenn ein Tatzeuge ihn bei einer Gegenüberstellung unter anderen Personen herausfinden kann. Aber unter wie vielen anderen muss der Zeuge den Täter herausfinden können und wie ähnlich dürfen die Kandidaten einander sein? Wenn man Platon folgt, dürfen wir erst dann von Wiedererkennen sprechen, wenn der Augenzeuge „das Unterscheidende des Dinges“ aufgefasst hat, also ein distinktives Merkmal oder einen Merkmalskomplex, der die Person von allen anderen (wirklichen? möglichen?) Personen unterscheidet. Dazu ist ein Tatzeuge gewöhnlich nicht in der Lage und die Polizei verlangt es sicherheitshalber auch nicht von ihm. Die Polizei ist zufrieden, wenn der Zeuge den Täter unter sechs anderen Personen „,identifizieren“ kann, nicht unter sieben Milliarden.

Platons Beispiel ist auch deshalb interessant, weil Menschen unter optimalen Bedingungen außerordentlich gut in Gesichtserkennung sind. Einige Menschen - vielleicht die eigenen Eltern - könnten wir vermutlich unter allen lebenden Menschen herausfinden, wenn wir genug Zeit hätten. ${ }^{7}$ Für den Fall der Gesichtserkennung scheint also Platons Standard der Distinktivität im Prinzip erfüllbar zu sein. Diesen Standard legen allerdings weder die Polizei noch die Gerichte an. Wenn sie es täten, könnte man eine Verurteilung

\footnotetext{
6 Sokrates wendet unter anderem ein, dass die geforderte distinktive Erklärung ja auf eine Erkenntnis der individuellen Verschiedenheit hinauslaufe, was die Definition von epistêmê zirkulär mache (Theaitet 209d-210a).

7 Diese erstaunliche Diskriminationsfähigkeit scheint auf der Wahrnehmung von Gestaltqualitäten zu beruhen. Menschen müssen Gesichter nicht wie eine Gesichtserkennungssoftware auf einzelne Merkmale abscannen.
} 
kaum jemals auf die Aussage von Augenzeugen stützen. ${ }^{8}$ Das In dubio pro reo-Prinzip verlangt ja, dass das Gericht nur verurteilt, wenn es keinerlei Zweifel an der Schuld des Angeklagten hat. Über mögliche Zweifelsgründe und über den Standard für deren Ausräumung schweigt sich die deutsche Strafprozessordnung aus. Es gilt der Grundsatz der „freien Beweiswürdigung", welcher besagt, dass es keine festen Regeln gibt, nach denen der Richter die Beweismittel zu würdigen hat.

Das Beispiel ist insofern noch aus einem weiteren Grund erkenntnistheoretisch interessant: Die Schuld des Angeklagten muss über jeden Zweifel hinaus erwiesen sein, aber der böse Dämon stellt vor Gericht keinen zu berücksichtigenden Zweifelsgrund dar. Man stelle sich einen Strafverteidiger vor, der nach Abschluss der Beweisaufnahme in seinem Schlussplädoyer die Möglichkeit des bösen Dämons ins Spiel brächte. Ein solcher Verteidiger würde keine Zweifel säen, sondern sich lächerlich machen. ${ }^{9}$ Obwohl die Rechtfertigungsstandards im Gerichtssaal weitaus höher sind als im Alltag, sind sie niedriger als im Descartes-Seminar und auch niedriger, als der Schluss des Theaitet nahelegt. Auf diese faktische Variabilität von Rechtfertigungsstandards reagiert bekanntlich der kontextualistische Wissensbegriff.

\section{ERste BeObachtung: Konsens ÜBER GeTtIERfäLle, KeIN KON- SENS ÜBER DIE ANALYSE DES WISSENSBEGRIFFS}

Meine Diskussion der Frage, was Gettierfälle uns über das Verhältnis von Begriffsanalysen und sprachlichen Intuitionen lehren, beginne ich mit zwei Beobachtungen, an die sich jeweils eine Frage anschließt.

\footnotetext{
${ }^{8}$ Vielleicht wäre das in Anbetracht der notorischen Unzuverlässigkeit von Zeugenaussagen auch besser so. Bei Gegenüberstellungen wird nicht selten diejenige Person als Täter benannt, die ihm am ähnlichsten sieht. Aus Wiederaufnahmeverfahren in den USA ist gut dokumentiert, dass der größte Teil der Fehlurteile auf falsche Zeugenaussagen zurückgeht. Geschworene neigen dazu, einem Augenzeugen auch dann Glauben zu schenken, wenn seine Aussage starken anderen Indizien widerspricht.

${ }^{9}$ Er würde sich übrigens nicht lächerlich machen, wenn er eine mögliche Gettierkomplikation erwähnte. Auch wenn in der rechtstheoretischen Literatur keine Einigkeit darüber besteht, ob für einen Beweis im juristischen Sinn - also für die Feststellung einer Tatsache durch richterliche Überzeugung - Wissen erforderlich ist oder etwas Schwächeres, wäre ein mit einer Gettierkomplikation behafteter Tatnachweis rechtsstaatlich inakzeptabel (vgl. dazu Pardo 2010). Schon Platon illustriert den Fall der bloß zufällig wahren Meinung mit einem forensischen Beispiel (Theaitet 201 b-c). Es kann also keine Rede davon sein, dass, wie manchmal behauptet wird, die Immunisierung des Wissensbegriffs gegen Gettierfälle allein für professionelle Erkenntnistheoretiker von Interesse wäre.
} 
Erste Beobachtung: Es gibt unter kompetenten Sprechern beträchtliche Einmütigkeit in der Beurteilung paradigmatischer Gettierfälle dass sie nämlich als Fälle von Nichtwissen zu klassifizieren sind. Ungleich weniger Einigkeit besteht hinsichtlich der Frage, auf welche Weise die klassische Wissensanalyse angesichts von Gettierfällen verbessert werden kann.

An diese Beobachtung schließt sich die Frage an, warum es offenbar so schwierig ist, konvergierende sprachliche Intuitionen in eine systematisch verbesserte Analyse des Wissensbegriffs zu überführen. - In der Beobachtung ist von paradigmatischen Gettierfällen, von Einmütigkeit der Beurteilung und von kompetenten Sprechern die Rede. Die damit verbundenen Annahmen sind erläuterungsbedürftig. Von paradigmatischen Gettierfällen spreche ich, weil es plausiblerweise auch Grenzfälle von Gettierfällen gibt. Sobald man Beispielfälle in der Grenzfallregion präsentiert, ist zu erwarten, dass auch unter kompetenten Sprechern die Einmütigkeit der Beurteilung schwindet. Von den drei Komponenten der klassischen Wissensanalyse sind zwei klarerweise gradierbar: Eine Überzeugung kann stärker oder schwächer, eine Rechtfertigung besser oder schlechter sein. Manche Philosophen nehmen an, dass sich neben der Rechtfertigungsqualität und der Überzeugungsstärke auch die Wahrheit abstufen lässt; Aristoteles und Frege hielten dies mit guten Gründen für absurd.

Beschränken wir uns auf die Dimension der Rechtfertigungsqualität, weil sie für das Gettierproblem am einschlägigsten ist. Wenn Gerechtfertigtsein gradierbar ist, lautet die naheliegende Frage, wie gut eine Rechtfertigung sein muss, um aus wahrer Meinung Wissen zu machen. Hier sind wiederum diejenigen Fälle am einschlägigsten, in denen die Gettierkomplikation ${ }^{10}$ selbst gradierbar oder sogar kontinuierlich skalierbar ist und der Grad des Gerechtfertigtseins entlang dieser Skala variiert. Betrachten wir Chisholms Schaf-Hund-Beispiel (vgl. Chisholm 1989, 93): Auf einer Wiese stehen ein Hund, der einem Schaf täuschend ähnlich sieht, sowie ein echtes Schaf, das hinter einem Busch verborgen ist. Der Betrachter blickt auf den Hund und kommt zu der Überzeugung, dass auf der Wiese ein Schaf steht. Die Überzeugung ist wahr, einen guten Grund hat der Betrachter auch: Was wie ein Schaf aussieht, ist in der Regel eins. Gleichwohl besteht Einigkeit darüber, dass er nicht weiß, dass auf der Wiese ein Schaf steht.

Nun kann man das Szenario schrittweise variieren, indem man die Anzahl der Schafe und Hunde und/oder deren Sichtbarkeit für den Betrachter ändert: Wie verhält es sich, wenn beide Tiere im Blickfeld des Betrachters stehen

\footnotetext{
${ }^{10}$ Den Ausdruck „Gettierkomplikation“ verwende ich zur Bezeichnung desjenigen Situationsmerkmals, das in Gettierfällen jeweils dafür verantwortlich ist, dass die wahre Überzeugung kein Wissen ist.
} 
und sein Blick auf den Hund fällt? Wie verhält es sich, wenn beide Tiere in seinem Blickfeld stehen und sein Blick auf das echte Schaf fällt? ${ }^{11}$ Wie verhält es sich, wenn der Hund hinter einem Busch verborgen ist? Wie verhält es sich, wenn sich hundert Schafe und nur ein einziger Hund auf der Wiese befinden? ${ }^{12}$ Hier eröffnet sich ein weites Betätigungsfeld für experimentelle Philosophen. Ich habe meine Studierenden gelegentlich über diese Variationen abstimmen lassen. Etliche Studierende beeindruckt der Umstand der subjektiven Ununterscheidbarkeit von Schafen und Hunden ganz unabhängig von Anzahl und Sichtbarkeit. In der letzten Variation befindet sich überhaupt kein Hund mehr auf der Wiese. Wenn aber einer da wäre, könnte ich ihn nicht von einem Schaf unterscheiden. Stets lautet die Frage: Weiß ich, dass auf der Wiese ein Schaf steht? Auch wenn die negativen Voten abnehmen, gibt es stets einige Studierende, die auch bei der letzten Variation noch für Nichtwissen plädieren: Die bloße Möglichkeit, sich zu täuschen, weil man das Schaf nicht von einem Hund unterscheiden könnte, sei mit Wissen unvereinbar. Dieser Auffassung liegt ein infallibilistischer Wissensbegriff zugrunde, nach dem Wissen nicht bloß eine wahre gerechtfertigte Überzeugung erfordert, sondern eine wahrheitsgarantierende Rechtfertigung. Vertreter eines infallibilistischen Wissensbegriffs nehmen aufgrund dieser verschärften Wissensdefinition sowie der Zusatzannahme, dass Menschen nicht über wahrheitsgarantierende Rechtfertigungsverfahren verfügen, in der Regel skeptische Positionen ein. Wenn schon die bloße Möglichkeit einer nichtentdeckbaren Täuschung den Wissensstatus gefährdet, ist kaum zu begründen, dass Menschen überhaupt etwas wissen.

Es gibt noch weitere für das Gettierproblem relevante Abstufungen der Rechtfertigungsqualität. So werfen Fälle, in denen sich eine Überzeugung auf mehrere voneinander unabhängige Rechtfertigungsquellen stützt, die Frage auf, ob nicht trotz der Gettierkomplikation Art und Umfang der anderen, nicht von der Komplikation betroffenen Belege für Wissen hinreichend sind. Beispielsweise kann sich bei inferentiell gewonnenen Überzeugungen der wissensunterminierende Zufall allmählich „verdünnen“, indem sein relativer Beitrag zur Rechtfertigung des Wissenssubjekts mit der schrittweisen Variation des Szenarios immer geringer wird. ${ }^{13}$

\footnotetext{
${ }^{11}$ Derartige Fälle nennt man „,unpossessed defeater“-Fälle. Ob sie als Gettierfälle zu klassifizieren sind, ist umstritten.

${ }^{12}$ Ähnlich lässt sich Goldmans Gettierfall der Scheunenattrappen (vgl. Goldman 1976, 772-3) variieren, was in der Literatur auch verschiedentlich unternommen worden ist, z. B. von Lackey, Comesaña, Neta/Rohrbaugh und Peacocke.

${ }^{13}$ Vgl. die Diskussion von Variationen des Gettierfalls der stehengebliebenen Uhr bei Baumann (2013)
} 
In Fällen, in denen die Gettierkomplikation kontinuierlich abstufbar ist, lassen sich die entsprechenden Szenarien in Soritesreihen anordnen. Gerechtfertigtsein und Gettierizität werden dann zu vagen Begriffen und damit zu Anwendungsfällen des Sorites-Paradoxons: Es ließe sich dann durch soritesförmige Argumentationen „beweisen“, dass auch klare Gettierfälle Fälle von Wissen sind.

Dass in meiner „ersten Beobachtung“ von paradigmatischen Gettierfällen die Rede ist, hat die Funktion, mir diese Komplikationen vom Leibe zu halten. Leider wird das unleugbare Phänomen der semantischen Vagheit nicht selten zum Anlass eines theoretischen Defätismus genommen, durch den man sich der weiteren Analysearbeit enthoben glaubt. Auch in der Diskussion des Gettierproblems gibt es diese Tendenz: Die Bemerkung, dass die Begriffe der Rechtfertigung und des Wissens eben vage seien, wird als Diskussionsstopper eingesetzt.

Die Gettierdebatte wird durch die Vagheitsdiagnose auf ein falsches Gleis geführt. Wenn man bei der Verfeinerung der vierten Bedingung, beispielsweise in elaborierten Antizufallstheorien, zunehmend in der Grenzfallregion landet, also bei der Frage, ob es sich überhaupt noch um einen Gettierfall handelt, ist dies ein Indiz für einen Themenwechsel. Man ist dann mit der Frage befasst, wie sich Wissensphänomene durch eine quantitative Bestimmung der erforderlichen Rechtfertigungsqualität präzise abgrenzen lassen. Diese Frage ist mit dem Gettierproblem nur lose verbunden. Die zentrale Herausforderung des Gettierproblems besteht in der Frage, was die Intuition, dass es sich nicht um Wissen handelt, in klaren Gettierfällen erklärt. Diese Herausforderung besteht auch dann, wenn „Gerechtfertigtsein“ und vielleicht sogar „Wissen“ randbereichsunscharf sind. ${ }^{14}$ Die Aufgabe, ein trennscharfes, Grenzfälle ausschließendes Kriterium für „Wissen“ $z$ u finden, ist gegenüber der Gettierherausforderung ein - oft unbemerkter - Themenwechsel.

Lässt man Grenz- und Zweifelsfälle von Gettierfällen beiseite, so ist die Konstellation die folgende: Auf der einen Seite gibt es eine philosophische Standarddefinition oder -analyse von Wissen, die seit zweieinhalbtausend Jahren nicht substantiell verbessert worden ist. Auf der anderen Seite gibt es spätestens seit Gettier eine offene, ständig wachsende Liste von Beispielfällen, die jeder kompetente Sprecher ohne besondere Ausbildung als Fälle von Nichtwissen klassifiziert, obwohl sie den Wortlaut der Standarddefinition erfüllen oder zu erfüllen scheinen. Der Bezug auf die Kompetenz gewöhnlicher Sprecher ist deshalb wichtig, weil Gettiers Entdeckung oder Wiederentdeckung ja keinen neuen Beurteilungsstandard ins Spiel bringt. Kompe-

\footnotetext{
${ }^{14}$ Hetherington (2001) vertritt die Auffassung, dass nicht nur Rechtfertigung, sondern Wissen selbst ein gradierbares Phänomen sei. Gettierfälle klassifiziert er als Fälle von „schlechtem“ Wissen, das sehr leicht kein Wissen hätte sein können.
} 
tente Sprecher waren auch zuvor schon disponiert, Gettierfälle, wenn sie ihnen denn präsentiert worden wären, als Fälle von Nichtwissen zu klassifizieren. In diesem Sinn stellt Jackson $(1998,36)$ richtig fest: ,[Gettier made] explicit what had been implicit in our classificatory practice all along". Alles spricht dafür, dass Gettier-Intuitionen zur Kompetenz gewöhnlicher Sprecher hinsichtlich des korrekten Gebrauchs des Verbs „wissen“ gehören.

Wenn nun paradigmatische Gettierfälle sowohl von Philosophen als auch von Nichtphilosophen einmütig als Fälle von Nichtwissen klassifiziert werden, erscheint die Erwartung legitim, dass sich diese Einmütigkeit auch in eine entsprechend verbesserte Wissensdefinition ummünzen lässt, die Gettierfälle ausschließt. Diese Erwartung ist aber in der seit fünf Jahrzehnten andauernden Gettierdebatte enttäuscht worden. Nagel bilanziert: „The resilience of the Gettier problem suggests that it is difficult (if not impossible) to develop any explicit reductive theory of knowledge that fully captures our actual patterns of response to particular examples" (Nagel 2012, 501). Dieser Befund ist freilich selbst erklärungsbedürftig. Warum lassen sich die konvergierenden Intuitionen nicht zu einer konsensfähigen Verbesserung der Wissensdefinition nutzen, die unserer tatsächlichen Praxis der Beurteilung von Gettierfällen entspricht? Schon dass die Aufgabe derart schwierig sein soll, erscheint rätselhaft.

\section{ZWEITE BEOBACHTUNG: BEGRIFFSANALYSEN WERDEN ANHAND VON INTUITIONEN ÜBER EINZELFÄLLE GETESTET}

Meine zweite Beobachtung betrifft nicht allein die Gettierdebatte, sondern die generelle Struktur philosophischer Debatten über vorgeschlagene Begriffsanalysen.

Zweite Beobachtung: Philosophen testen die Angemessenheit von vorgeschlagenen Analysen oder Explikationen philosophischer Grundbegriffe häufig anhand von Gegenbeispielen, die in Form sprachlicher Intuitionen über Einzelfälle präsentiert werden („,method of cases“). In diesen Gegenbeispielen liegt entweder das Phänomen vor, ohne dass das Analysans erfüllt ist, oder vice versa, wie in den Gettierfällen.

Dass sprachliche Intuitionen über Einzelfälle als Testfälle für die Angemessenheit einer Begriffsanalyse verwendet werden, versteht sich nicht von selbst. Die Konstellation, dass die beste verfügbare philosophische Analyse eines Begriffs und die voranalytischen Intuitionen kompetenter Sprecher über die korrekte Anwendung dieses Begriffs auf einzelne Fälle einander widersprechen, lässt ja mehrere Reaktionen zu. Warum verlassen wir uns in solchen dialektischen Kontexten meist auf unsere Intuitionen und versuchen, 
die Analyse anzupassen? Wir könnten ja auch umgekehrt die Analyse verwenden, um unsere sprachlichen Intuitionen zu belehren. Aber dies geschieht in der Gettierdebatte nicht. ${ }^{15}$ Es geschieht auch in früheren Stadien der Analyse des Wissensbegriffs nicht, wenn - wie im Theaitet - dem Überzeugtsein die Bedingungen der Wahrheit und des Gerechtfertigtseins hinzugefügt werden. Zur Wahrheit: Dass „wissen“ ein faktives Verb ist - „S weiß, dass p“ impliziert „,p“ -, ist ein linguistischer Befund und keine erkenntnistheoretische These. Zum Gerechtfertigtsein: Wenn jemand auf die Frage nach der Anzahl der Teller im Küchenschrank eine Antwort gibt, die sich beim Nachzählen als wahr herausstellt, hat er mit der Rückfrage zu rechnen „Wusstest Du es oder hast Du geraten?" Diese Rückfrage ist jedem kompetenten Sprecher verständlich. Zu seiner Sprachkompetenz gehört auch, dass er die Antwort durch die Rechtfertigungsbedingung restringiert weiß. Solche Dialoge belegen, dass schon der philosophisch unbelehrte Alltagsbegriff des Wissens über den einer wahren Meinung hinausgeht.

Die Konstellation, dass vortheoretische Intuitionen als Testfälle genutzt werden, liegt auch in anderen philosophischen Debatten vor. Dazu zwei Beispiele: Das Sinnkriterium im Logischen Empirismus sollte Sinn von Unsinn scheiden. Schlick, Carnap und Ayer hatten das Kriterium aufgestellt, dass alle sinnvollen Aussagen durch Rückführung auf Protokollsätze empirisch überprüfbar sein müssen (ich gehe über Unterschiede bedenkenlos hinweg). Bald wurde deutlich, dass unter anderem Naturgesetze und Dispositionsaussagen den Verifikationstest nicht bestehen. Erklärte man dem Sinnkriterium folgend diese Sätze für sinnlos? Nein, man versuchte, das Kriterium anzupassen, auf dass sich diejenigen Sätze als sinnvoll erweisen mochten, die nicht den Eindruck erweckten, unsinnig oder kognitiv gehaltlos zu sein und an denen den Debattenteilnehmern gelegen war. Offenkundig hatten sie Intuitionen über sprachlichen Sinn und kognitiven Gehalt, die vom Sinnkriterium unabhängig waren.

Auch in der Kausalitätstheorie spielen sprachliche Intuitionen über Einzelfälle eine prominente Rolle. So werden die verschiedenen Versionen der kontrafaktischen Theorie der Kausalität typischerweise anhand von Gegenbeispielen getestet (,preemption“, „,overdetermination“). Es werden Fälle angeführt, in denen wir einen singulären Kausalsatz für wahr halten, die vor-

15 „If anything, the moral of the Gettier discussion is that the reverse is often true: explicit analyses are themselves dependent on a priori intuitions concerning specific cases, or equivalently, on a priori intuitions about certain conditionals. The Gettier literature shows repeatedly that explicit analyses are hostage to specific counterexamples, where these counterexamples involve a priori intuitions about hypothetical cases." (Chalmers/Jackson 2001, 321-2) Eine seltene Ausnahme ist Weatherson (2003), der die klassische Analyse des Wissensbegriffs für besser gestützt hält als Intuitonen über vermeintliche Gegenbeispiele. 
geschlagene Analyse ihn aber für falsch erklärt, oder in denen umgekehrt die Analyse uns ein Kausalurteil aufzwingt, das wir intuitiv nicht zu fällen geneigt sind. Mithilfe der Gegenbeispiele wird die Angemessenheit der vorgeschlagenen Analyse bestritten, woraufhin die Verfechter der herausgeforderten Theorie versuchen, ihre Analyse derart zu verfeinern, dass auch die als Gegenbeispiele präsentierten Fälle erklärt werden können.

Was lehrt uns der Umstand, dass philosophische Theorien und Begriffsanalysen anhand von faktischen oder vorgestellten Gegenbeispielen überprüft werden? Zunächst dokumentiert er einen Unterschied in der epistemischen Gewissheit: Sprecher sind sich der sprachlichen Intuitionen, die der korrekten Verwendung bestimmter Ausdrücke in Einzelfällen zugrunde liegen, offenbar sicherer als der Angemessenheit von philosophischen Analysen und Theorien, die diese Intuitionen explizieren und präzisieren sollen. Nur so lässt sich erklären, dass die Einzelfälle überhaupt als Testfälle akzeptiert werden. Andernfalls könnten sich die Vertreter der angegriffenen Theorien zurücklehnen und erklären: „Ich habe Dir doch gerade definiert, was Kausalität ist, was Wissen ist und was sprachlicher Sinn ist. Nun kommst Du mit Deinen anekdotischen Gegenbeispielen. Was heißt hier überhaupt ,Gegenbeispiel'? Gegen Definitionen gibt es keine Gegenbeispiele. Definitionen mögen mehr oder weniger zweckmäßig sein, aber sie sind qua Definitionen weder wahr noch falsch, können mithin auch nicht durch Gegenbeispiele falsifiziert werden." Diese Reaktion findet man aber in den erwähnten Debatten nicht. Vielmehr werden die Gegenbeispiele als Herausforderung begriffen.

Diese Struktur der Debatten dürfte sich daraus erklären, dass eine stillschweigende Übereinkunft darüber besteht, dass man im Bereich der Grundbegriffe unserer deskriptiven Metaphysik nicht stipulativ, sondern explikativ verfahren sollte, also mit dem Anspruch, denjenigen Begriff zu erläutern, den wir tatsächlich leidlich erfolgreich und - hoffentlich - konsistent verwenden. Dass man in diesen Kontexten eher von „Begriffsanalysen“ oder „Begriffsexplikationen“ spricht als von „Definitionen“, ist ein Indiz für diese Übereinkunft. Man akzeptiert die Auflage der „deskriptiven Adäquatheit“, derzufolge die Extensionen von Analysans und Analysandum sich voneinander nicht zu stark unterscheiden dürfen. Unter dieser Auflage steht auch die Gettierdebatte: Eine verbesserte Analyse des Wissensbegriffs hat nicht nur Gettierfälle auszuschließen, sondern soll sich zudem am Ziel der deskriptiven Adäquatheit des Analysans oder Explikans orientieren und diese erhöhen. 


\section{WAS SIND „INTUITIONEN“ ÜBER GETTIERFÄLLE?}

Das beschriebene Testen von Begriffsanalysen anhand von Intuitionen über Einzelfälle wurde praktiziert, lange bevor dieses Vorgehen in der Metaphilosophie als ,method of cases“ bezeichnet und auf seinen Wert und seine Beweiskraft befragt wurde. Angesichts der umfangreichen und verzweigten jüngeren Debatte über Intuitionen in der Philosophie erscheint es angezeigt, diejenige Art von Intuitionen, auf die sich die Beurteilung von Gettierfällen stützt, genauer zu charakterisieren.

Hinter der verbreiteten Berufung auf Intuitionen verbirgt sich eine Reihe von unterschiedlichen Phänomenen. Oftmals hat die Redeeinleitung ,intuitively speaking" bloß die Funktion eines sogenannten Heckenausdrucks (,device of hedging“, vgl. Cappelen 2012, 36-9), mit dem der Sprecher den erhobenen Geltungsanspruch abschwächt und sich gerade nicht auf eine besondere Erkenntnisquelle beruft. Einige Teilnehmer der Debatte verwenden „Intuition“ für ein besonderes Vermögen der rationalen, nichtsinnlichen Anschauung oder für den die Ausübung des Vermögens begleitenden mentalen Zustand. Für Bealer sind Intuitionen ,intellectual seemings” hinsichtlich des Wahrheitswerts von Propositionen, die weder auf sinnliche noch introspektive Anschauungen zurückgehen, sondern ,sui generis“ sind (vgl. Bealer 1996, 123; 1998, 207-8). Manche Autoren behaupten, dass mit Intuitionen eine besondere Phänomenologie des Als-wahr-Erscheinens verbunden sei. Ein Hauptstrang der Debatte kreist um die Frage, ob Intuitionen a priori sind, ein weiterer um die Frage, wie zuverlässig oder fehlbar sie als Erkenntnisquelle sind. In den letzteren Kontext gehört auch die Debatte über die interkulturelle Variabilität von Intuitionen. Für einige experimentelle Philosophen beweist Einmütigkeit über Gettierfälle unter westlichen Erkenntnistheoretikern nicht viel, denn ,philosophers risk mistaking consensus for correctness when weighing the evidential value of their intuitions" (Nagel 2012, 496).

Um in diese Debatten nicht tiefer einsteigen zu müssen als nötig, müssen wir diejenige Art von Intuitionen identifizieren, die für die Beurteilung von Gettierfällen zentral ist. Wohlan: Es geht in der Gettierdebatte um begriffliche, sprachliche oder metasprachliche Intuitionen, die in Urteile darüber münden, ob bestimmte tatsächliche oder hypothetische Einzelfälle unter die Extension der natürlichsprachlichen Prädikate „Wissen“, „knowledge“ oder „epistêmê" fallen, bzw. unter die entsprechenden Begriffe. ${ }^{16}$ Goldman nennt

\footnotetext{
${ }^{16}$ Den Unterschied zwischen Prädikaten (sprachlichen Ausdrücken) und Begriffen vernachlässige ich in diesem Beitrag oft. Der Unterschied kann relevant werden, wenn es um die behauptete interkulturelle Variabilität von Intuitionen über Gettierfälle geht: In Sprecherbefragungen wird die Verwendung sprachlicher Ausdrücke ermittelt, was aber nicht eo ipso einen Schluss auf unterschiedliche Begriffe zulässt.
} 
diese Intuitionen „classification or application intuitions, because they are intuitions about how cases are to be classified" (Goldman 2007, 4). ${ }^{17}$ Hinsichtlich ihres Inhalts hat man sie als ,epistemische Intuitionen“ bezeichnet (z. B. Nagel 2007), da sie die Anwendung epistemischer Begriffe betreffen. Die Quelle dieser Intuitionen ist plausiblerweise die gewöhnliche Sprachkompetenz kompetenter Sprecher einer natürlichen Sprache. Das Adjektiv „intuitiv“ bezeichnet primär die Art des Zugriffs des Sprechers auf Elemente seiner Sprachkompetenz, die als „,vortheoretisch“, „unreflektiert", „direkt“, „sicher" und „schnell“ beschrieben wird. Die subjektive Sicherheit und die Geschwindigkeit des Zugriffs dürften sich unter anderem aus der Gebrauchsfrequenz erklären: „Know” ist das achthäufigste Verb im Englischen. Sprecher müssen sich häufig und schnell zwischen ,know“ und konkurrierenden Verben entscheiden und gewinnen deshalb eine hohe intuitive Sicherheit im Gebrauch. $^{18}$

Meine tentative Bestimmung der fraglichen Intuitionsart lautet also:

Die in der Beurteilung von Gettierfällen konsultierten „Intuitionen“ sind vortheoretische metasprachliche Überzeugungen oder Urteile über die Korrektheit der Anwendung sprachlicher Ausdrücke auf hypothetische oder tatsächliche Fälle. In diesen Urteilen greifen Sprecher punktuell auf ein implizites Wissen von Elementen der eigenen Sprachkompetenz zu.

Ich schließe sechs Bemerkungen zur Verortung dieser Intuitionsart in der metaphilosophischen Debatte über Intuitionen an:

(1) Die Frage, ob die fraglichen Wahrheiten a priori oder a posteriori erkannt werden, ist zu krude, um für die Charakterisierung von Anwendungsintuitionen hilfreich zu sein. ${ }^{19}$ Durch den Bezug auf die Sprachkompetenz mag sich der Streit über den apriorischen Charakter sprachlicher Intuitionen entschärfen lassen: Die Aprioristen betonen, dass es zur Beurteilung neben dem Besitz des Begriffs keiner empirischen Information bedarf und dass auch Gettiers Argument ein apriorisches sei, das eine apriorische Wahrheit

\footnotetext{
${ }^{17} \mathrm{Ob}$ man diese Klassifikationsintuitionen als sprachliche oder als metasprachliche ansieht, scheint für die Gettierdebatte nicht besonders relevant zu sein. Angesichts eines Gettierfalls lautet das objektsprachliche Urteil: „S weiß nicht, dass p“, das metasprachliche: „Dies ist kein Anwendungsfall des Ausdrucks ,Wissen“".

${ }^{18}$ „Further evidence for the intuitive character of knowledge recognition could be found in the speed and frequency of our real-time decisions between verbs of thinking and knowing - 'knows' and 'thinks' are both heavily used, ranking at \#8 and \#12 of the Oxford English Corpus list of our most vommon verbs. Judgments naturally become intuitive when made very frequently." (Nagel 2012, 501)

${ }^{19}$ Vgl. zu dieser Kritik Williamson 2007, 165-9 und 189-195.
} 
ans Licht gebracht habe. ${ }^{20}$ Sie abstrahieren davon, dass der Erwerb und die Beherrschung eines Begriffs empirische Phänomene sind, dass die kompetente Anwendung des Verbs „wissen“ mit der empirischen Kenntnis des Sprachgebrauchs in einer Sprachgemeinschaft verwoben ist oder sogar darauf superveniert und dass die Feststellung der Unterschiede in den möglichen Anwendungsfällen von „Wissen“, anders als bei logischen und mathematischen Begriffen, ebenfalls empirische Feststellungen erfordert.

(2) Der Bezug auf die Sprachkompetenz erklärt zwanglos sowohl die relative Zuverlässigkeit von Anwendungsintuitionen als auch deren Grenzen: Die Kompetenz keines empirischen Sprechers ist mit der von Chomsky fingierten idealen Sprecher/Hörer-Kompetenz identisch. Außerdem ist zum ausgefransten Rand der Extension hin ein Nachlassen sowohl der Zuverlässigkeit als auch der subjektiven Sicherheit des Urteils zu erwarten. In allzu exotischen Fällen gibt es nicht einmal ein fact of the matter bezüglich der korrekten Anwendung eines natürlichsprachlichen Ausdrucks. ${ }^{21}$ Darum ist auch die Frage, womit Wissen in allen möglichen Welten identisch ist, eine schlechte Frage.

(3) Die reklamierte besondere Phänomenologie des Als-wahr-Erscheinens scheint für sprachliche Intuitionen keine wesentliche Rolle zu spielen. Einschlägiger ist die „opaqueness-of-origin phenomenology“ von Intuitionen (Goldman 2007, 11), die sich auf den Umstand zurückführen lässt, dass Sprecher kein explizites Wissen über die Genese der meisten Elemente ihrer Sprachkompetenz besitzen. Dieser Umstand hilft auch zu verstehen, was sprachliche Intuitionen von einem rein intellektuellen Vermögen unterscheidet: ,the process of generating classification intuitions has more in common with memory retrieval than with purely intellectual thought or ratiocination" (ebd., 20). Introspektive Transparenz des Ursprungs ist für die Verwendung sprachlicher Intuitionen in der „method of cases“ auch nicht erforderlich, da die Details des individuellen kindlichen Spracherwerbs für den Korrektheitsstandard, den kompetente Sprecher internalisiert haben, irrelevant sind.

(4) Dass es sich um einen kompetenzbasierten punktuellen Zugriff auf Intuitionen über Einzelfälle handelt, schließt bestimmte platonistische Auffassungen wie diejenige von Sosa aus, dass der Gegenstand einer Intuition eine „abstract proposition“ sei (Sosa 1998, 263-4). Abstrakte Propositionen bestimmt Sosa als solche, die ,abstract away from any mention of particulars“

\footnotetext{
${ }^{20}$, ,Gettier's argument was an a priori argument, in which empirical information played no essential role, and its conclusion is a paradigmatic example of a non-obvious a priori truth" (Chalmers/Jackson 2001, 321).

${ }^{21}$ „Nur in normalen Fällen ist der Gebrauch der Worte uns klar vorgezeichnet; wir wissen, haben keinen Zweifel, was wir in diesem oder jenem Fall zu sagen haben. Je abnormaler der Fall, desto zweifelhafter wird es, was wir nun hier sagen sollen.“ (Wittgenstein, PU § 142)
} 
(ebd., 258). Diese Bestimmung passt nicht zu gettierfallbezogenen Intuitionen und allgemein nicht zur ,method of cases“, für die der Bezug auf Einzelfälle wesentlich ist. ${ }^{22}$

(5) Das Zustandekommen von Urteilen angesichts von Gettierfällen lässt sich ohne großen Verlust unter Verzicht auf den Ausdruck „Intuition“ ausdrücken. Die drei Champions der Philosophie der normalen Sprache - Wittgenstein, Ryle und Austin - sind in ihren begriffsbezogenen Untersuchungen ohne diesen Ausdruck ausgekommen. Austin hat ihn zugunsten der ,what we should say when"-Methode vermieden.

(6) Ein Verzicht auf die Rede von Intuitionen hätte den Vorteil, dass der normative Aspekt der Berufung auf sprachliche Richtigkeit klarer $\mathrm{zu}$ Tage tritt. Es geht ja in der Gettierdebatte um die korrekte Verwendung eines Ausdrucks. Die Quelle der Normativität ist in etwas anderem zu suchen als in den mentalen Zuständen von Sprechern, denn der Standard der Korrektheit für die Anwendung eines Ausdrucks ist kein psychologischer.

Devitt argumentiert, dass Intuitionen nur die Daten liefern, aber nicht über deren Validität entscheiden. ${ }^{23}$ Sprachliche Intuitionen seien weder a priori noch besonders verlässlich, vielmehr seien sie ,empirical theory-laden central-processor responses to phenomena, differing from many other such responses only in being fairly immediate and unreflective, based on little if any conscious reasoning" (Devitt 2006, 103). Intuitionen besäßen allenfalls indirekte Beweiskraft. Deshalb plädiert Devitt dafür, statt der Intuitionen die direkte Evidenz für sprachliche Korrektheit zu untersuchen, die er in der sprachlichen Wirklichkeit verortet, insbesondere im tatsächlichen Sprachgebrauch. Dieser lasse sich am besten durch linguistische Korpusanalysen untersuchen. $^{24}$

Ich halte die antimentalistische Stoßrichtung von Devitts Kritik für berechtigt, werde aber aus Gründen der Darstellungsökonomie die Rede von sprachlichen Intuitionen beibehalten. Konvergierende sprachliche Intuitionen supervenieren plausiblerweise auf semantische oder soziale Tatsachen - auf eine etablierte Klassifikationspraxis oder auf implizite Bedeutungs- oder Verwendungsregeln. Diese Tatsachen oder Regeln müssen freilich psycholo-

\footnotetext{
${ }^{22}$ So auch Goldman: „[Sosa's] definition threatens to exclude our primary philosophical examples, viz., application intuitions. These often concern particulars, both particular individuals and particular situations“ (Goldman 2007, 10).

${ }^{23}$ „Although a speaker's competence in a language obviously gives her ready access to the data of that language, the data that the intuitions are about, it does not give her ready access to the truth about the data." (Devitt 2013, 269)

${ }^{24}$ „In particular, philosophers should seek evidence in usage. They should seek direct evidence in linguistic reality itself rather than simply relying on the indirect evidence of intuitions about that reality. A major source of such evidence is the corpus, the linguistic sounds and inscriptions that the folk have produced and are producing as they go about their lives.“ (Devitt 2013, 271)
} 
gisch realisiert sein, weil andernfalls nicht zu erklären wäre, dass Sprecher das Prädikat „wissen“ auf unbekannte Fälle projizieren können, wovon Gettierfälle Beispiele geben.

\section{BEGRIFFSANALYSE UND DIE „METHOD OF CASES“}

Da das Hauptinteresse dieses Beitrags der Frage gilt, was Gettierfälle uns über das Verhältnis von sprachlichen Intuitionen und Begriffsanalysen lehren, ist nun ein Verständnis von Begriffsanalyse gesucht, das den beschriebenen Eigenarten sprachlicher Intuitionen und ihrer Verwendung in der ,,method of cases" Rechnung trägt. In der Geschichte der analytischen Philosophie ist das Verfahren der Begriffsanalyse (conceptual analysis) auf unterschiedliche Weisen bestimmt worden. ${ }^{25}$ Ein der „method of cases“ kongenialer Vorschlag stammt von Grice:

I hope that it will now be fairly clear what sort of thing I mean by "conceptual analysis." To be looking for a conceptual analysis of a given expression $E$ is to be in a position to apply or withhold $E$ in particular cases, but to be looking for a general characterization of the type of cases in which one would apply $E$ rather than withhold it. And we may notice that in reaching one's conceptual analysis of $E$, one makes use of one's ability to apply and withhold $E$, for the characteristic procedure is to think up a possible general characterization of one's use of $E$ and then to test it by trying to find or imagine a particular situation which fits the suggested characterization and yet would not be a situation in which one would apply E. [...] [O]ne could not test a suggested characterization in this way, unless one relied on one's ability to apply or withhold $E$ in particular cases. (Grice 1958, 174)

Die entscheidende Gegenüberstellung ist die zwischen „being in a position“ und „looking for" im zweiten Satz des Zitats, in dem der Ausgangspunkt und das Ziel des Unternehmens unterschieden werden: Kompetente Sprecher sind schon in der Lage, den Ausdruck richtig auf einzelne Fälle anzuwenden, gesucht ist eine allgemeine Charakterisierung des Typs von Fällen, auf die der Ausdruck zutrifft. Den Ausgangspunkt der Begriffsanalyse bilden kompetenzbasierte sprachliche Intuitionen über Einzelfälle, das Ziel ist die Beantwortung der Frage nach dem Begriff oder der Natur des fraglichen Phänomens. ${ }^{26}$ Erklärungsbedürftig ist, warum die allgemeine Charakterisie-

${ }^{25}$ Ich erinnere an die Prozess/Produkt-Ambiguität von „Begriffsanalyse“. Im Folgenden geht es primär um das Verfahren der Begriffsanalyse und nur sekundär um das Ergebnis.

${ }^{26}$ „Philosophical analysis, of course, doesn't simply aim to answer questions about particular cases. Epistemology isn't much interested in whether this or that example is an instance of knowledge; 
rung des Phänomens des Wissens so viel schwieriger ist als die Beurteilung von Einzelfällen.

Eine instruktive Parallele zu dieser Asymmetrie bietet das Recht mit seinem spannungsvollen Verhältnis zwischen Gesetzgebung und Rechtsanwendung. Dem Gesetzgeber stellt sich die Aufgabe, eine allgemeine Beschreibung eines Tatbestandstyps zu formulieren, die Einzelfälle subsumieren soll, deren faktische Details dem Gesetzgeber noch unbekannt sind, weil sie in der Zukunft liegen. Der Rechtsanwender hat dann die tatsächlichen Einzelfälle unter den Gesetzestext zu subsumieren. Von diesen Subsumtionen sind viele trivial, aber es kommen auch Fälle mit Eigenarten vor, an die der Gesetzgeber nicht gedacht hat, die im Gesetzestext weder explizit ein- noch ausgeschlossen sind und für die das Gericht gleichwohl entscheiden muss, ob der Fall unter den im Gesetz beschriebenen Tatbestand fällt oder nicht. Für rechtlich zulässige Gesetzesauslegungen gilt die sogenannte Wortlautgrenze; eine Entscheidung contra legem, die sich über den Wortlaut des Gesetzes hinwegsetzt, kann nicht mehr als Auslegung gelten. Manche der „teleologischen“ Auslegungen der Gerichte bewegen sich so hart an der Wortlautgrenze, dass der Gesetzgeber sich zur Reaktion gezwungen sieht, also zur Schließung der Gesetzeslücke oder allgemein zur Präzisierung der Rechtsnorm. Dabei hat der Gesetzgeber mit einer ähnlichen Asymmetrie zu kämpfen wie der Erkenntnistheoretiker, der auf dem Wege der „method of cases“ seine Analyse des Wissensbegriffs zu verbessern sucht. Auch im Recht ist es leichter, eine Gesetzeslücke zu finden als sie zu schließen ${ }^{27}$ - so wie es allgemein leichter ist, Gegenbeispiele zu ersinnen als allgemeine Formulierungen zu finden, die alle Gegenbeispiele ausschließen. Salopp gesprochen: Mit Gegenbeispielen torpediert ein Narr mehr Begriffsanalysen, als zehn Weise reparieren können.

Wenn nun die Frage lautet, ob im Konfliktfall sprachliche Intuitionen über Einzelfälle stets Begriffsanalysen trumpfen, dann ist zunächst festzuhalten, dass dies ein Kampf unter Ungleichen ist. Die Gegenüberstellung von Intuition und Analyse übersieht einen bedeutsamen Umstand, der in der Gettierdebatte selten reflektiert wird: Unsere sprachlichen Intuitionen zu Gettierfällen befragen wir stets ex post, also im Lichte einer vorliegenden oder jedenfalls spezifizierten Gettierkomplikation. Damit nehmen wir bei der Beurteilung des Gettierfalls eine Ressource in Anspruch, die bei Aufstellen der allgemeinen Wissensdefinition nicht verfügbar war. Ex ante lautet die Analyse: „Fälle, die die Bedingungen J, T und B erfüllen, sind Wissen“. Dann tritt ein Fall ein, der die Bedingungen erfüllt, aber auf eine so bizarre Weise, dass wir ihn intuitiv nicht als einen Fall von Wissen akzeptieren. Unsere Gettier-

rather, it aims to say what knowledge is in general [...]. Individual cases are typically introduced as test cases of one or more general accounts." (Sosa 2007, 6)

${ }^{27}$ Für eine originelle rechtstheoretische Diskussion dieses Problems vgl. Katz 2011, 69-136. 
Intuition hat den Inhalt: „Dies ist ein Fall von wahrer, gerechtfertigter Überzeugung, die kein Wissen ist". Da nun in paradigmatischen Gettierfällen diese Intuition nicht schwach und idiosynkratisch ist, sondern stark und weithin geteilt, scheint unsere Beurteilungskompetenz Elemente zu enthalten, die durch die klassische Analyse des Wissensbegriffs nicht ausgeschöpft werden. Dies ist nur deshalb möglich, weil die Analyse, also die generische Charakterisierung eines Phänomentyps, in charakteristischer Weise unterbestimmt war.

\section{7. „LEERSTELLEN“ UND „BLINDE FLECKEN“}

Um dieses Unterbestimmtsein genauer zu fassen, möchte ich einen Terminus aus der Literaturtheorie ins Spiel bringen. Als „Leerstellen“ oder „Unbestimmtheitsstellen“ bezeichnet man in der Narratologie Bestimmungslücken in einem fiktionalen Text. Sie finden sich überall dort, „wo man auf Grund der im Werk auftretenden Sätze von einem bestimmten Gegenstand (oder von einer gegenständlichen Situation) nicht sagen kann, ob er eine bestimmte Eigenschaft besitzt oder nicht" (Ingarden 1968, 49). ${ }^{28}$ So ist beispielsweise die Frage, ob Emma Bovary ein Muttermal auf der rechten Schulter hat, eine gegenstandslose Frage, wenn der Text sich darüber ausschweigt. Eine Romanfigur ist nicht genauer bestimmt als sie durch den Romantext bestimmt wird. In der wirklichen Welt gibt es keine solchen Bestimmungslücken. Anders als fiktionale Charaktere und Situationen ist die wirkliche Welt auch an ihren nichtrepräsentierten Stellen auf eine bestimmte Weise beschaffen, die andere Beschaffenheiten ausschließt. Man kann das so ausdrücken, dass die wirkliche Welt ontisch dichter ist als unsere sprachlichen und mentalen Repräsentationen der Welt. Die ontische Dichte ist für die deskriptive Unerschöpflichkeit der Welt und ihrer konkreten Einzeldinge verantwortlich: In jeder noch so detaillierten Beschreibung wirklicher Einzeldinge, Ereignisse und Situationen bleiben vorhandene Eigenschaften unbeschrieben (vgl. dazu Keil 2005). Wie Austin gesagt hat: „Fact is richer than diction. ${ }^{، 29}$

Da ein tatsächliches Geschehen ontisch dichter ist als unsere Repräsentationen, wird eine generische Beschreibung eines Situationstyps stets Leerstellen aufweisen, die durch Wirkliches auf unterschiedliche Weise aufgefüllt

\footnotetext{
${ }^{28}$ Ingarden spricht von „Unbestimmtheitsstellen“. Den Ausdruck „Leerstelle“ hat Wolfgang Iser eingeführt und mit rezeptionstheoretischen Zusatzannahmen darüber verbunden, in welcher Weise der Leser während der Lektüre solche Leerstellen „auffüllt“. Ich verwende im Folgenden den Ausdruck „Leerstelle“ zur Bezeichnung dessen, was Ingarden „Unbestimmtheitsstelle“ nennt.

${ }^{29},[\mathrm{H}]$ owever well equipped our language, it can never be forearmed against all possible cases that may arise and call for description: fact is richer than diction." (Austin 1956, 195)
} 
werden können, ohne die Beschreibung falsch zu machen. Wenn die Leerstellen auf „normale“ oder erwartbare Weise aufgefüllt sind, tritt uns der Dichteunterschied gar nicht ins Bewusstsein. Wenn sie auf irreguläre Weise aufgefüllt werden, können zum Beispiel Gettierfälle entstehen oder „abweichende Kausalketten“, die Gettierfälle der analytischen Handlungstheorie. Die Ausdrücke „normal“ und ,irregulär“ erklären freilich nichts; ein allgemeines Unterscheidungskriterium für normale und irreguläre Auffüllungen wäre vielmehr ein wichtiger Baustein für eine Lösung des Gettierproblems.

Der narratologische Begriff der Leerstelle betrifft das Verhältnis einer sprachlichen Repräsentation zum Repräsentierten. Als sein erkenntnistheoretisches oder psychologisches Pendant kann man das Phänomen des blinden Flecks ansehen. Erkenntnistheoretisch interessant sind ja insbesondere die unbemerkten Leerstellen einer sprachlichen oder mentalen Repräsentation. $\mathrm{Zu}$ denjenigen Situationsmerkmalen, die sich im blinden Fleck oder im toten Winkel des epistemischen Subjekts befinden, kann es sich gar nicht erst eine Überzeugung bilden. ${ }^{30}$ Deshalb sind auf blinde Flecken zurückgehende Fehlannahmen so tückisch. ${ }^{31}$ Mit der Rede von blinden Flecken meint man in der Regel nicht vermeidbare Fehler, sondern den Sachverhalt, dass bestimmte Dinge einfach nicht ins Blickfeld des epistemischen Subjekts geraten, weil es ohne Hilfe nicht darauf kommen kann, an sie zu denken und deshalb auch keine epistemische Pflicht dazu hat. Der Rat: „Achte auf das, was in Deinem blinden Fleck liegt!“ ist wenig hilfreich, denn um ihn zu befolgen, bräuchte man zumindest geeignete metakognitive Strategien.

Bevor ich das Gesagte auf das Gettierproblem anwende, möchte ich das Phänomen des blinden Flecks durch ein Beispiel illustrieren.

\section{EIN BEISPIEL: DAS „PHANTOM VON HEILBRONN“}

Das sogenannte Phantom von Heilbronn war eine unbekannte weibliche Person (Polizeijargon: UWP), der zwischen 1993 und 2009 eine Vielzahl von Straftaten zugeordnet wurden, weil man ihre DNA-Spuren an den Tatorten fand. Es ging um mehr als 40 Taten, die an verschiedenen Orten in einem Radius von 400 Kilometern begangen wurden: Gartenhaus- und Büroeinbrüche, Diebstähle, Fahrzeugaufbrüche sowie mehrere Tötungsdelikte. Nach

\footnotetext{
${ }^{30}$ Der blinde Fleck im Gesichtsfeld kommt physiologisch dadurch zustande, dass sich an der Stelle der Netzhaut, an der der Sehnerv austritt, keine Lichtrezeptoren befinden.

${ }^{31}$ Aus einem Vorwort von Ernst Tugendhat: ,[I] ch habe weder einer Lebensgefährtin für ihre Opferbereitschaft zu danken noch zu versichern, daß alle verbleibenden Fehler nur mir allein zuzuschreiben sind. Es sind nicht Fehler, die einen erschrecken, sondern Blindheit, die blinden Flecken." (Tugendhat 2007, 9)
} 
dem Mord an einer Polizistin in Heilbronn 2007 wurden die Ermittlungen intensiviert. Über die Jahre beschäftigte der Fall fünf Sonderkommissionen und sechs Staatsanwaltschaften in Baden-Württemberg, Rheinland-Pfalz, dem Saarland und Österreich. Man suchte, so die Presse, nach „einer cleveren Mörderin [...], die das gesamte Establishment, die Polizei-Intelligenz, die Kriminaltechnik und ihre hochsensible Analysetechnik narrt [...], der es scheinbar egal ist, ob man sie findet, so dilettantisch und fahrlässig, wie sie ihre Spuren hinterlässt“ “. ${ }^{32}$ Die Ermittlungsbehörden zogen viele Hypothesen in Betracht, auch einen gerissenen Täter, der fremde Spuren legt, sowie Fehler bei der Entnahme der DNA-Proben an den Tatorten oder bei deren Auswertung im Labor. Eines Tages fand man die ominöse DNA-Spur am Tatort einer tödlichen Schlägerei, an der nachweislich nur männliche Personen beteiligt waren, so dass man noch einmal neu ermittelte und 2009 endlich auf die Verursacherin der DNA-Spur kam: Es war keine Serientäterin, sondern eine Mitarbeiterin eines oberfränkischen Verpackungsbetriebs, welcher alle beteiligten Landeskriminalämter mit Abstrichbestecken belieferte.

An diese Möglichkeit einer vorherigen Kontamination sämtlicher für die Spurensicherung verwendeten Wattestäbchen durch ein und dieselbe Person hatte niemand gedacht. Auf diese Erklärung muss man erst einmal kommen, und die fünf Sonderkommissionen sind nicht darauf gekommen, wiewohl ja Hypothesen über Verfahrensunsicherheiten - Verunreinigungen während der Entnahme oder bei der Auswertung im Labor - in Betracht gezogen worden waren. Die Komplikation befand sich im blinden Fleck aller Ermittlungsbehörden.

Der Fall des Phantoms von Heilbronn illustriert das Phänomen des blinden Flecks, doch was hat er mit dem Gettierproblem zu tun? Bisher nicht viel, denn es handelt sich ja nicht um einen Gettierfall. Aus seinen Ingredienzen lässt sich indes ein Gettierfall konstruieren - wenn man nämlich annimmt, dass die Spurenverursacherin die Taten tatsächlich begangen hat:

Helga B. konfektioniert Wattestäbchen in einem oberfränkischen Verpackungsbetrieb. Die Firma liefert Abstrichbestecke an mehrere süddeutsche Landeskriminalämter. Aber Helga B. hat ein dunkles Geheimnis. Nach der Arbeit fährt sie oft ziellos durch die Gegend, bricht hier in eine Gartenlaube ein, stiehlt dort ein Motorrad und tötet eines Tages sogar eine Polizistin, in der sie ihre verhasste Stiefschwester zu erkennen glaubt. Über die Jahre kommen mehr als 40 Taten zusammen. Dabei hat Helga B. sorgfältig darauf geachtet, an keinem Tatort Spuren zu hinterlassen. Eine große Hilfe waren ihr dabei die Plastikhauben und Schutzhandschuhe, die sie von ihrem Arbeitsplatz hat mit${ }^{32}$ Christian Schüle, „Das Unfassbare“, DIE ZEIT 2. 4. 2009, Nr. 15, http://www.zeit.de/2009/15/M-
Phantom. 
gehen lassen, wo sie seit Jahren unbenutzt herumliegen. Endlich entschließt sich die Sonderkommission zum Phantom von Heilbronn aus lauter Verzweiflung zum größten Massenscreening der deutschen Kriminalgeschichte. Auch Helga B. muss aufgrund ihres Wohnortes eine Speichelprobe abgeben und wird einige Tage später in Anwesenheit von zwölf Fernsehteams verhaftet. Im Polizeipräsidium knallen die Sektkorken. Helga B. kann sich nicht erklären, wie ihre Spuren an die Tatorte gekommen sind und leugnet die Taten.

Die Gettierfrage lautet natürlich: Weiß die Polizei, dass Helga B. die Straftaten begangen hat? Über weitere Beweise verfügt die Polizei nicht, aber der genetische Fingerabdruck ist als Beweismittel zugelassen. Einen genetisch identischen eineiigen Zwilling hat Helga B. nicht.

Es wäre eine eigene Untersuchung wert, der Frage nachzugehen, welche Lösungsvorschläge für das Gettierproblem in der Lage sind, diesen Gettierfall aus dem Wissensbegriff auszuschließen. Ich vermute, dass auch elaborierte Safety accounts dazu nicht oder nur um einen unangemessen hohen Preis in der Lage sind. Safety-Theorien bestimmen Wissen, grob gesprochen, als wahre Überzeugung, die nicht leicht hätte falsch sein können (Pritchard, Sosa, Williamson, Brendel, Grundmann). Es ist mit guten Gründen vorgeschlagen worden, die Forderung nach epistemischer Sicherheit nicht auf die Überzeugungen des Wissenssubjekts, sondern auf die verwendeten Methoden zu beziehen: Um Wissen handle es sich nur, wenn die verwendete Methode in allen nahen möglichen Welten nicht zu einer falschen Überzeugug führt. ${ }^{33}$

Es ist schwer zu sehen, auf welche Weise der Ansatz der epistemischen Methodensicherheit diesen Gettierfall ausschließen könnte. Die wahre Überzeugung, dass Helga B. die Taten begangen hat, hätte nicht leicht falsch sein können, denn die verwendete Methode des genetischen Fingerabdrucks hat ein hohes Maß an Zuverlässigkeit. Freilich hängt die Einschätzung der Reliabilität des Verfahrens davon ab, welche möglichen Welten man zum Vergleich in Betracht zieht. Ist eine mögliche Welt ohne Vorabkontamination von Abstrichbestecken der wirklichen Welt ähnlicher als eine Welt mit bei den Taten hinterlassenen DNA-Spuren? Anhand welcher Gesichtspunkte soll man das entscheiden? Müsste man dafür die objektive Wahrscheinlichkeit

\footnotetext{
${ }^{33}$ „Damit eine wahre Überzeugung zu Wissen führt, muss die Methode, d. h. die Art und Weise, mittels derer S zur wahren Überzeugung in der aktualen Welt gelangt ist, auch in allen nahen möglichen Welten zu Überzeugungen führen, die ebenfalls wahr sind.“ (Brendel 2013, 63) Als „Wissenstheorien epistemischer Methodensicherheit“ bezeichnet Brendel Theorien, „die eine wahre Überzeugung nur dann als Wissen auszeichnen, wenn die Methode der Überzeugungsgewinnung auch epistemisch sicher ist" (ebd.).
} 
der Fehlerquelle im Heilbronner Fall einschätzen $?^{34} \mathrm{Zu}$ den anderen in Betracht gezogenen Fehlerquellen bei der Anwendung des Verfahrens des genetischen Fingerabdrucks liegen systematische Untersuchungen vor: Eine mit der Qualitätskontrolle des Verfahrens beauftragte forensische Arbeitsgruppe hat Fehlerquoten zwischen $0,4 \%$ und $0,7 \%$ ermittelt. Die Fehler gingen vornehmlich auf eine Vertauschung von Proben und auf falsches manuelles Übertragen von Ergebnissen zurück. ${ }^{35}$

Ein Verteidiger der durch den Reliabilismus inspirierten Theorie der epistemischen Methodensicherheit könnte argumentieren, man müsse das im Heilbronner Fall verwendete Verfahren im Lichte der nun bekannten Fehlerquelle spezifischer charakterisieren, nämlich als Sicherung von DNA-Spuren mittels verunreinigter Abstrichbestecke. Ein solches Verfahren ist freilich zur Ermittlung oder Überführung von Tätern alles andere als verlässlich. Aber welchen Wert hätte es für eine Safety-Theorie des Wissens, ex post derartige „Methoden“ zu fingieren? Mit gleichem Recht könnte man die in Russells bekanntem Beispiel verwendete Methode zur Feststellung der Uhrzeit als „Blick auf eine Uhr, die unbemerkt stehengeblieben ist“" charakterisieren. ${ }^{36}$

Methoden zur Generierung von Wissen sollten für Wissenssubjekte anwendbare Methoden sein. Dies gilt umso mehr, wenn die Safety-Theorie, wie bei Sosa, mit einer tugenderkenntnistheoretischen Auffassung kombiniert wird. Tugenden sollen schließlich erwerbbar und ihre Ausübung zurechenbar sein. Das scheint nicht möglich, wenn epistemische Tugenden ihrerseits externalistisch durch einen Faktor individuiert werden, den die Person nicht unter Kontrolle hat, nämlich das faktische Treffen der Wahrheit selbst unter unglücklichen Umständen. ${ }^{37}$

Was verlässlichkeitsherabsetzende Komplikationen betrifft, die im blinden Fleck der Wissenssubjekte liegen, so lässt sich das abstrakte Wissen von dieser Möglichkeit nicht zur Charakterisierung von Methoden verwenden, deren Anwendung Gettierfälle ausschließt, auch wenn sich ex post in Kenntnis der faktischen Gettierkomplikation stets eine „Methode“ konstruieren lassen mag, durch die der Fehler vermieden worden wäre. Eine Theorie der episte-

\footnotetext{
${ }^{34}$ In der Literatur zu den Safety-Theorien gibt es eine Diskussion darüber, ob die geforderte epistemische Sicherheit mit objektiver Wahrscheinlichkeit korreliert ist oder nicht; vgl. z. B. Williamson 2009.

${ }^{35}$ Vgl. S. Rand, M. Schürenkamp, C. Hohoff, B. Brinkmann: „The GEDNAP Blind Trial Concept Part II. Trends and Developments“, International Journal of Legal Medicine 118.2 (2004), 83-89.

${ }^{36}$ Brendel nimmt diese Konsequenz in Kauf. Hinsichtlich des Beispiels des defekten Thermometers, das heimlich auf die korrekte Temperatur eingestellt wurde, argumentiert sie, ,dass wir das Konsultieren eines defekten Thermometers üblicherweise nicht als verlässliche Methode zur Gewinnung wahrer Überzeugungen über die Raumtemperatur ansehen würden“ (Brendel 2013, 65).

${ }^{37}$ Sosa selbst bestimmt eine intellektuelle Tugend als „, quality bound to help maximize one's surplus of truth over error" (Sosa 1991, 225).
} 
mischen Methodensicherheit überstrapaziert den Begriff der Methode, wenn sie jeden Fall von unvorhersehbarem epistemischem Pech nachträglich zu einem Defekt der verwendeten Methode ummünzt oder, in der tugenderkenntnistheoretischen Variante, messerscharf schließt, dass im Falle eines unglücklichen Fehlgehens die „truth-tracking ability“ des Subjekts nicht eingesetzt worden ist.

Ich kritisiere wohlgemerkt nicht, dass in reliabilistischen Theorien die Verlässlichkeit einer Methode nicht aus der beschränkten Perspektive des Wissenssubjekts bestimmt wird, sondern externalistisch. Entscheidend ist vielmehr, dass die Anwendung von im allgemeinen zuverlässigen Methoden im Einzelfall stets zu falschen Ergebnissen führen kann. Dies lässt sich nicht ausschließen, solange Menschen nicht über wahrheitsgarantierende Überprüfungsverfahren verfügen. Nach allem, was wir wissen, verfügen sie nicht über solche, mithin können Wahrsein und bestens begründetes Fürwahrhalten auseinanderklaffen. (Vertreter eines epistemischen Wahrheitsbegriffs bestreiten das.) Auch bei der Anwendung hochzuverlässiger Methoden kann man epistemisches Pech verschiedener Art haben, und bei bestimmten Arten von Pech kommt es zu Gettierfällen. Die einzige Möglichkeit, zu wahrheitsgarantierenden Methoden zu gelangen, dürfte sein, sie zirkulär als solche zu definieren.

\section{EINE THESE: GETTIERKOMPLIKATIONEN NISTEN IN BLINDEN FLECKEN}

Den Exkurs über Leerstellen und blinde Flecken lasse ich in eine substantielle These zum Gettierproblem münden, die ein charakteristisches Merkmal von Gettierfällen betrifft. Die These lautet, dass diejenigen Komplikationen, die einen Gettierfall zu einem solchen machen, sich stets in den blinden Flecken der Situationsrepräsentation des epistemischen Subjekts befinden, dessen Wissensstatus in Frage steht. Gettierfälle sind Fälle, in denen die Welt einen Weg gefunden hat, ihre Komplikationen in die blinden Flecken der Annahmen des Wissenssubjekts zu platzieren bzw. - semantisch statt psychologisch ausgedrückt - in die Leerstellen seiner Situationsbeschreibungen. ${ }^{38}$ Am Beispiel von vier klassischen Gettierfällen: Es ist der Person

\footnotetext{
${ }^{38}$ In Williamsons Rekonstruktion der Gettier-Intuition mithilfe kontrafaktischer Konditionale gibt es eine Überlegung, die eine gewisse Verwandtschaft mit der hier vorgetragenen aufweist. Williamson argumentiert, dass das Konditional „Notwendigerweise gilt: Wenn jemand in einer Relation zu einer Proposition steht, wie sie in einer bestimmten Gettierfall-Beschreibung vorliegt, hat er eine gerechtfertigte, wahre Überzeugung, die kein Wissen ist“ ein zu starker Ausdruck der Gettier-Intuition wäre. Weil Gettierszenarien mannigfach unterbestimmt seien, sei es stets möglich, die fehlenden Details durch Hintergrundannahmen zu ergänzen, die zur Folge haben, dass das Subjekt keine
} 
schlicht nicht in den Sinn gekommen, dass die Uhr vor exakt 24 Stunden stehengeblieben sein könnte, dass sie selbst genauso viele Münzen in der Tasche haben könnte wie der erfolgreiche Mitbewerber, dass sie in der Umgebung mit perfekten Scheunenattrappen zu rechnen hat oder dass es auf der Wiese Hunde gibt, die von Schafen ununterscheidbar sind.

In den verbreiteten Formulierungen, dass in Gettierfällen „zufällig“, „glücklich“ oder „auf unzuverlässige Weise“ eine gerechtfertigte wahre Überzeugung erworben wird, bleibt dieser Umstand unterbelichtet. Es genügt für Gettierfälle nicht, dass die Überzeugung glücklich oder durch ein wenig verlässliches Verfahren zustande gekommen ist, vielmehr ist es wesentlich, dass dem epistemischen Subjekt die Gettierkomplikation in charakteristischer Weise verborgen war.

Warum soll das so sein? Warum könnte dem Subjekt nicht schon vor Erheben des Wissensanspruchs oder vor einer Evaluation seines Wissensstatus die mögliche Gettierkomplikation präsent gewesen sein? Nehmen wir an, sie wäre ihm präsent gewesen. Es gibt dann zwei Möglichkeiten: Entweder wäre das Subjekt zu einer zutreffenden Überzeugung hinsichtlich des fraglichen Situationsmerkmals gekommen oder zu einer unzutreffenden. Spielen wir die beiden Fälle anhand des Uhrenbeispiels ${ }^{39}$ durch: Ich blicke auf meine Uhr, sie zeigt 17.25 Uhr, ich rechne aber aus irgendeinem Grund damit, dass sie stehengeblieben sein könnte.

(i) Ich überprüfe die Uhr und finde meine Vermutung bestätigt. Damit ist meine Rechtfertigung für das Kennen der Uhrzeit zerstört. Es gelingt mir deshalb nicht mehr, davon überzeugt zu sein, dass es $17.25 \mathrm{Uhr}$ ist, a fortiori weiß ich es nicht.

(ii) Ich überprüfe die Uhr, bin aber dabei zu nachlässig. Ich gelange zu der falschen Überzeugung, dass meine Uhr funktioniert und zuverlässig die Zeit anzeigt. Da diese Überzeugung die einzige ist, auf die sich mein Wissensanspruch stützt, ist die Bedingung des Gerechtfertigtseins nicht erfüllt, also handelt es sich nicht um einen Gettierfall. ${ }^{40}$

Rechtfertigung besitzt (vgl. Williamson 2007, 185). Angemessener sei die Rekonstruktion der Gettier-Intuition durch das kontingente kontrafaktische Konditional „Wenn jemand eine bestimmte Gettierfall-Beschreibung erfüllte, hätte er eine gerechtfertigte, wahre Überzeugung, die kein Wissen wäre“, deren Vorteil Williamson so beschreibt: „By using the counterfactual conditional, we in effect leave the world to fill in the details of the story, rather than to do it all ourselves" (ebd., 186). Ob die kontrafaktische Formulierung das beschriebene Problem löst, ist umstritten, denn sie müsste ja ausschließen, dass die wirkliche Welt die Details auf eine Weise auffüllt, die die Rechtfertigung unterminiert und das Konditional falsch macht. Zu dieser Debatte vgl. Ichikawa (2009).

${ }^{39} \mathrm{Vgl}$. Russell 1948, 113 und 170.

${ }^{40}$ Genauer könnte man die Rechtfertigungsbasis als zweigeteilte beschreiben. Zum einen habe ich eine Wahrnehmungsüberzeugung hinsichtlich der Zeigerstellung, zum zweiten eine explizite An- 
In beiden Fällen, (i) und (ii), lässt sich mithin mit Bezug auf die klassische Analyse angeben, warum es sich nicht um einen Fall von Wissen handelt. Dies ist das gewünschte Ergebnis: Sobald die Gettierkomplikation erwogen wird und das epistemische Subjekt sich eine Meinung zu ihr bildet, sei sie zutreffend oder nicht, wird die Rechtfertigung auf die eine oder andere Weise unterminiert, mithin handelt es sich nicht mehr um einen Gettierfall.

Die These, dass Gettierkomplikationen sich stets in den blinden Flecken der Situationsrepräsentation des Wissenssubjekts verbergen, müsste man an vielen weiteren Beispielen durchspielen, um sie generalisieren zu können. Dabei würden sich vermutlich interessante Fallunterscheidungen ergeben. Ich habe vier klassische Beispiele erwähnt, die die These stützen. Auch in den Gettierfällen der Handlungstheorie - den abweichenden Kausalketten, die die Angemessenheit der Handlungsdefinition der kausalen Handlungstheorie bedrohen - funktioniert die These gut. ${ }^{41}$

Dass Gettierkomplikationen in blinden Flecken nisten, erklärt zum einen, warum die Rechtfertigung aus der Perspektive des Erkenntnissubjekts gar keinen Defekt aufweist. Es hat nach bestem Wissen und Gewissen geurteilt und hatte fraglos eine subjektive Rechtfertigung für seinen Wissensanspruch. Es hat keine andere Methode angewandt als die, die sich in zahllosen Fällen als zuverlässig erwiesen hat. Gewöhnlich verschwenden Personen beim Blick auf eine Uhr keinen Gedanken darauf, ob die Uhr funktioniert. Eine Überprüfung der Funktionstüchtigkeit verlangt man ihnen im Normalfall auch nicht ab, sie gehört nicht zu ihren epistemischen Pflichten. Dies dürfte seinen Grund darin haben, dass heutige Quarzuhren einen hohen Grad von Zuverlässigkeit und Ganggenauigkeit aufweisen. Wenn aber aufgrund eines begründeten Zweifels eine Überzeugung hinsichtlich der Funktionsfähigkeit erworben wird, wie im Fall (ii), muss sie zutreffend sein, damit die Rechtfertigungsbedingung erfüllt ist.

Ist die These, dass Gettierkomplikationen in blinden Flecken nisten, originell? War nicht in der Beschreibung von Gettierfällen immer schon impliziert, dass das Subjekt von der Gettierkomplikation nichts weiß? Nun, die These vom blinden Fleck ist spezifischer. Es genügt für einen Gettierfall nicht, dass das Subjekt von der Komplikation nichts weiß. Wenn es ein offenkundiges Situationsmerkmal vorwerfbar ignoriert, also seine epistemischen Pflichten verletzt, wäre die Qualität seiner Rechtfertigung stark beeinträchtigt. Hätte es zum Beispiel klare Indizien für die Unzuverlässigkeit der Uhr gegeben, die das Subjekt missachtet hat - so kündigen viele Quarzuhren das Versagen der Batterie durch ein anormales Verhalten des Sekundenzei-

nahme hinsichtlich der Funktionstüchtigkeit der Uhr. Wenn die letztere Annahme irrig ist und die erstere zur Rechtfertigung nicht ausreicht, bin ich nicht gerechtfertigt.

${ }^{41}$ Vgl. dazu Keil 2007, bes. 86. 
gers an -, dann wäre es in der Überzeugung, die Uhrzeit zu kennen, nicht oder nur sehr schwach gerechtfertigt, so dass es sich nicht mehr um einen Gettierfall handelte. ${ }^{42}$

Das beschriebene Merkmal von Gettierfällen macht auch verständlich, warum die Ex ante- und die Ex post-Beurteilung divergieren: Sobald die Leerstelle der Situationsbeschreibung in Kenntnis der eingetretenen Komplikation aufgefüllt wird, wird angebbar, auch für das Wissenssubjekt selbst, was an dieser Rechtfertigung in diesem besonderen Fall mangelhaft war, obwohl eine gleichlautende Rechtfertigung ohne das Vorhandensein der Gettierkomplikation gut genug gewesen wäre, um die wahre Überzeugung zu Wissen zu machen.

Wahre Überzeugungen, die das Subjekt in nichtvorwerfbarer Weise für Wissen hält, können sich als Fälle von Nichtwissen herausstellen. Der Wissenshintergrund, auf dem epistemische Subjekte ihre Überzeugungen rechtfertigen, hat also eine nicht-monotone Struktur: Rechtfertigungen sind nicht gegenüber Zusatzinformation immun. Hinzukommende Informationen können eine Rechtfertigung untergraben, obwohl sie mit dem Wortlaut der zuvor rechtfertigenden Überzeugungen verträglich sind. Die Leerstellentheorie bietet eine elegante Erklärung für die Eigenschaft der Nichtmonotonie. ${ }^{43}$

\section{TRUMPFEN INTUITIONEN BEGRIFFSANALYSEN?}

Damit komme ich auf meine Frage zurück, ob Gettierfälle eine prinzipielle Überlegenheit von sprachlichen Intuitionen über Einzelfälle gegenüber philosophischen Begriffsanalysen anzeigen.

${ }^{42}$ Zwischen dem fahrlässigen Ignorieren eines Indizes und dem schuldlosen Übersehen eines verborgenen Situationsmerkmals mag es fließende Übergänge geben. In der Grauzone hätte man es dann mit Grenzfällen von Gettierfällen zu tun.

${ }^{43}$ Das Auffüllen der Leerstelle lässt sich auch als das Sichtbarmachen zuvor verborgener Prämissen beschreiben: Im Fall des Heilbronner Phantoms wird von der wahren Prämisse „Wir haben bei allen Probenabstrichen an allen Tatorten die DNA von NN gefunden" auf die falsche Konklusion „Die UWP hat an allen Tatorten ihre DNA hinterlassen“ geschlossen, weil eine falsche implizite Prämisse über den kausalen Pfad zwischen Spurenverursacherin und DNA-Spur im Spiel war. Im Fall der stehengebliebenen Uhr wird das Subjekt erst durch die Gettierkomplikation darauf aufmerksam, dass das Urteil „Es ist 17.25 Uhr“ keine rein wahrnehmungsinduzierte Überzeugung ausdrückt, sondern als impliziter Schluss mithilfe einer versteckten Prämisse über die Funktionstüchtigkeit der Uhr rekonstruiert werden kann. - Die Interpolation von impliziten Prämissen in „wahrnehmungsnahen“ Gettierfällen (z. B. „Was wie ein Schaf aussieht, ist in der Regel auch eins") ist übrigens dazu angetan, den Unterschied zwischen inferentiellen und nichtinferentiellen Gettierfällen zu verwischen (vgl. dazu Bernecker 2013, $31 \mathrm{f}$ ). 
Die Antwort muss „Jein“ lauten. Bei näherer Betrachtung zeigt sich, dass nicht Intuitionen gegen Analysen stehen, sondern dass die entscheidende Asymmetrie eine andere ist. Was in Gettierfällen konfligiert, sind nicht Analyse und Intuition als solche, sondern die generische Charakterisierung eines Situationstyps und die Ex post-Beurteilung einer einzelnen Situation in Anbetracht einer bestimmten Gettierkomplikation. Damit nehmen wir bei der Beurteilung des Gettierfalls eine Ressource in Anspruch, die bei Aufstellen der allgemeinen Wissensdefinition nicht verfügbar war. Es herrscht in einer entscheidenden Hinsicht keine Waffengleichheit zwischen Intuition und Analyse: Wenn Subjekte angesichts von konkreten Gettierfällen ihre sprachliche Intuition befragen, können sie besondere Situationsmerkmale beurteilen, die in der allgemeinen Charakterisierung des Situationstyps nicht vorkamen. Was die Intuitionen überlegen macht, sind also die zusätzlichen Informationsressourcen, die beim Befragen der Intuition angezapft werden.

Der Umstand, dass Begriffsanalysen legitimerweise anhand von Intuitionen über Einzelfälle getestet werden, erweist deshalb keine Überlegenheit der Intuitionen im Sinne einer besseren Methode zur Erreichung desselben Ziels. Die Ziele sind von vornherein verschieden: Beim Aufstellen einer analytischen Wissensdefinition versucht man dem klassischen Verständnis von Begriffsanalyse zufolge, notwendige und gemeinsam hinreichende Bedingungen für Wissen anzugeben: „Fälle, die die Bedingungen J, T, B erfüllen, sind Fälle von Wissen“. In Gettierfällen sehen wir: nicht immer. Sprachliche Intuitionen spielen bei der Beurteilung von Gettierfällen eine destruktive Rolle, während die Analyse des Wissensbegriffs ein konstruktives Ziel hat.

Die Aufgabe, eine allgemeine Charakterisierung derjenigen Komplikationen zu geben, die mit Wissen unvereinbar sind, geht weit über die kompetente Beurteilung von Einzelfällen hinaus. Es wäre nicht fair und nicht weise, von der Analyse des Wissensbegriffs etwas zu verlangen, was sie nicht leisten kann. Eine Analyse des Wissensbegriffs kann nicht ans Licht bringen, wo Wissenssubjekte blinde Flecken haben, welche nichtrepräsentierten Situationsmerkmale sich in diesen blinden Flecken befinden mögen und welche dieser vielfältigen unbekannten Merkmale mit Wissen unvereinbar wären. Um den Ertrag der destruktiven ,method of cases“ konstruktiv zu verwerten, also in eine systematische Verbesserung der Wissensdefinition zu überführen, bedürfte es einer Typologie, einer Systematik oder einer vollständigen Disjunktion aller möglichen Gettierkomplikationen. Ich habe kein allgemeines Argument dafür, dass das am wenigsten ehrgeizige dieser Ziele - die Aufstellung einer Typologie - unerreichbar ist, nur eine Diagnose, warum es so schwierig zu erreichen ist.

Sprachliche Intuitionen über Einzelfälle fallen weder vom Himmel noch kommen sie aus dem Bauch. Sie sind Teil unserer erworbenen Sprachkom- 
petenz, supervenieren auf die tatsächliche Urteilspraxis in einer Sprachgemeinschaft und folgen den dieser Praxis impliziten Regeln. Allerdings ist die Praxis, auf die sie supervenieren, vielfältig und unübersichtlich und die Art der Supervenienz möglicherweise auch. Es ist, wie Wittgenstein sagt, „eine Hauptquelle unseres Unverständnisses, daß wir den Gebrauch unserer Wörter nicht übersehen. Unserer Grammatik fehlt es an Übersichtlichkeit" (PU § 122). Philosophische Begriffsanalysen stellen im Idealfall Übersicht her. Das Operieren mit sprachlichen Intuitionen über Einzelfälle hat eine andere, komplementäre Aufgabe. Gegenbeispiele stellen nicht Übersicht her, sondern erweisen trügerische Übersichtlichkeit als solche.

\section{FRAGEN UND ANTWORTEN}

Ich habe in diesem Beitrag keinen Vorschlag zur Lösung des Gettierproblems im Sinne einer verbesserten Analyse des Wissensbegriffs gemacht. Ich habe mich auch nicht an einer Bestimmung einer für Wissen hinreichenden Rechtfertigungsqualität versucht. Vielmehr habe ich ein gemeinsames strukturelles Merkmal von Gettierfällen herausgearbeitet, das uns zu verstehen hilft, warum sich sprachliche Intuitionen über Wissenszuschreibungen in Einzelfällen nicht in eine wasserdichte und gettierfallimmune Analyse des Wissensbegriffs überführen lassen. Abschließend möchte ich die skizzierte Überlegung mit einigen Einwänden und offenen Fragen konfrontieren.

(i) Die Einführung des Konzepts der „Leerstelle“ in die Gettierdebatte stellte darauf ab, dass die wirkliche Welt ontisch dichter ist als jede sprachliche Repräsentation der Welt und deshalb „deskriptiv unerschöpflich“. Die meisten Gettierfälle werden aber als hypothetische Fälle in Form von Gedankenexperimenten vorgestellt, in denen es gar keine verborgenen faktischen Details gibt.

Das ist richtig. Gettier selbst leitet seine beiden fiktiven Beispiele mit den Worten „Suppose that“ ein. Williamson $(2007,179-207)$ behandelt Gettierfälle als Gedankenexperimente und als Anwendungsfall kontrafaktischen Räsonierens. Unsere sprachlichen Intuitionen in Gettierfällen sind ganz unabhängig davon, ob der Fall wirklich oder hypothetisch ist. ${ }^{44}$ Unsere Beurteilung fiktiver Gettierfälle steht aber sehr wohl unter dem stillschweigenden Vorbehalt, dass der beschriebene Fall keine weiteren verborgenen für die

\footnotetext{
44 „For example, in the Gettier case, it is irrelevant whether or not John's case is actual: a subject can know that if John's case as described is actual, then John does not know that someone owns a Ford."(Chalmers/Jackson 2001, 324)
} 
Wissenszuschreibung relevanten Merkmale aufweist. In hypothetischen Szenarien kann man das stipulieren, in wirklichen Fällen kann man es niemals ausschließen.

Aus der Diskussion über andere Gedankenexperimente in der Philosophie ist bekannt, dass für die Sicherung der sprachlichen Intuitionen oft eine Anreicherung der Szenarien mit weiteren Details erforderlich ist. Wir behandeln die vorgestellten Fälle, $a l s o b$ sie wirkliche wären, führen uns die geschilderte Situation möglichst detailliert vor Augen und überlegen ,what we should say when"." 45

Der Bezug auf blinde Flecken spielt auch in fiktiven Gettierfällen eine Rolle. Die Gettierkomplikation verbirgt sich dort freilich nicht im blinden Fleck des Autors der Geschichte oder des Evaluierers, sondern des fingierten epistemischen Subjekts.

Vielleicht habe ich noch nicht hinreichend deutlich gemacht, dass der psychologische Begriff des blinden Flecks und der semantische Begriff der Leerstelle nicht genau dieselbe Rolle spielen: Blinde Flecken der epistemischen Subjekte sind ein Situationsmerkmal, das alle Gettierfälle, wirkliche wie hypothetische, gemeinsam haben. Der Bezug auf Leerstellen von Beschreibungen und die deskriptive Unerschöpflichkeit wirklicher Situationen erklärt, warum Intuitionen über Einzelfälle sich nicht in eine systematische Verbesserung der Analyse des Wissensbegriffs überführen lassen: Das wörtliche Zutreffen der Beschreibungen, die die hinreichenden Bedingungen für Wissen ausdrücken sollen, kann niemals garantieren, dass sich in den Leerstellen der Beschreibungen keine Gettierkomplikation verbirgt.

(ii) Es erscheint nach wie vor rätselhaft, warum konvergierende Intuitionen über Einzelfälle sich nicht zu einer schrittweisen Verbesserung der Analyse des Wissensbegriffs nutzen können sollten. Die „method of cases“ wird in anderen Fällen - Beispiel Kausalität - doch auch erfolgreich zu diesem Zweck eingesetzt.

Ich stelle nicht in Abrede, dass die Diskussion von Gettierfällen unsere Einsicht in die Natur des Wissens verbessert. Zum Beispiel lässt sich jeder einzelne unkontroverse Gettierfall induktiv zu einer zusätzlichen Ausschlussbedingung verallgemeinern. Was für Russells Uhr und für Pritchards Thermometer gilt, sollte allgemein für defekte Messinstrumente gelten, die zufälligerweise korrekt anzeigen. Solche Ausschlussbedingungen für Wissen sind aber weit von demjenigen Abstraktionsniveau entfernt, das für eine systema-

\footnotetext{
${ }^{45}$ Aus Austins Seminar wird berichtet, dass bei der Anwendung der ,what we sould say when“Methode die vorgestellte Situation immer genauer charakterisiert wurde und dass die sprachlichen Intuitionen der Diskutanten umso stärker konvergierten, je detaillierter die Beschreibung war (vgl. Urmson 1969, 84-5).
} 
tische Verbesserung der Wissensanalyse erforderlich wäre. Diejenigen Klauseln dagegen, die hinreichend allgemein formuliert sind, dass man sie als vierte Bedingung in die Wissensanalyse schreiben könnte - Antizufallsklauseln, no false lemmas, no defeaters, sensitivity, safety, etc. -, sind ausnahmslos von Gegenbeispielen betroffen, und zwar sowohl von solchen, die die Bedingungen als zu restriktiv erweisen, als auch von solchen, die sie als zu liberal erweisen. Jede bisher vorgeschlagene allgemeine Klausel ist anhand von Beispielfällen als überinklusiv, als unterinklusiv oder als beides kritisiert worden. Dies könnte schlicht bedeuten, dass Begriffsanalyse eben schwer ist. Ich vermute aber, dass die Sache schlimmer steht und dass Analyse und Gegenbeispiel sich bei der Wissensanalyse wie Hase und Igel zueinander verhalten.

Klassifiziert man den Fall mit der stehengebliebenen Uhr als Fall von Nichtwissen, so verpflichtet man sich darauf, alle hinreichend ähnlichen Fälle ebenfalls als Fälle von Nichtwissen zu klassifizieren. Nun müsste man nur noch „hinreichende Ähnlichkeit“ bestimmen, das für die Gettierkomplikation verantwortliche Situationsmerkmal spezifizieren und es zu einem Typus verallgemeinern. Dann müsste man mit den Gettierkomplikationen der anderen Beispielklassen ebenso verfahren und am Ende das Gelernte zu einer Klausel verallgemeinern, die als die gesuchte vierte Bedingung fungieren kann.

Ich kenne, um es zu wiederholen, kein Apriori-Argument dafür, dass dieses Verfahren nicht zum Ziel führen kann. Wenn die möglichen Gettierkomplikationen genügend gemeinsam haben, dass sie die Formulierung einer solchen Bedingung erlauben, die weder über- noch unterinklusiv ist (oder eine handhabbare Disjunktion solcher Bedingungen), dann wäre das Gettierproblem gelöst. $O b$ die Komplikationen dafür genügend gemeinsam haben, ist eine offene Frage. ${ }^{46}$ Gesucht ist ja eine verbesserte Analyse, die alle Gettierfälle ausschließt. Diese Analyse hat Bedingungen oder allgemeine Merkmale zu formulieren, die in jedem einzelnen Fall von Wissen vorliegen müssen oder die nicht vorliegen dürfen. Wenn aber das über Leerstellen jeder Beschreibung empirischer Phänomene Gesagte ${ }^{47}$ richtig ist, gibt es keine Garantie dafür, dass nicht der nächste Einzelfall, der die fraglichen Bedin-

\footnotetext{
${ }^{46}$ Chalmers und Jackson argumentieren, dass das Gettierproblem gezeigt habe, dass ,the complete set of sufficient conditions for knowledge is [...] indefinite and open-ended [...]; if it were not, we would have a satisfactory explicit analysis." (Chalmers/Jackson 2001, 322)

${ }^{47}$ Die Einschränkung auf empirische Phänomene ist wichtig. Rein begrifflich individuierte abstrakte Gegenstände sind nicht deskriptiv unerschöpflich (vgl. Keil 2005, 87-90). Es sind deshalb nicht alle Begriffsanalysen mit der beschriebenen Schwierigkeit behaftet, sondern nur diejenigen, die von empirischen Phänomenen erfüllt werden müssen. Der Begriff der Primzahl ist recht einfach zu analysieren und nicht für unabsehbare Gegenbeispiele anfällig.
} 
gungen Wort für Wort erfüllt, daneben weitere, „interstitielle“ Merkmale aufweist, die die Rechtfertigung unterminieren oder auf eine so bizarre Weise einrahmen, dass die Wissenszuschreibung zurückgezogen werden muss.

Es gibt übrigens eine verblüffend einfache sprachliche Möglichkeit, in blinden Flecken befindliche Gettierkomplikationen mitzubezeichnen, nämlich die demonstrative oder indexikalische Bezugnahme auf das betreffende Situationsmerkmal oder den fraglichen Gegenstand. Leider steht sie in Begriffsanalysen nicht zur Verfügung, da diese sich nach üblicher Auffassung auf deskriptive sprachliche Mittel beschränken müssen. Am Beispiel des Stellenbewerbers mit den zehn Münzen in der Tasche: Hätte Jones seine Überzeugung durch den von einer Zeigegeste begleiteten Satz „Dieser Mann da bekommt den Job" ausgedrückt, dann hätte er etwas Falsches gesagt und somit schon aus diesem Grunde kein Wissen ausgedrückt. Eine demonstrative Bezeichnung der gemeinten Person hätte den trügerischen Anschein des Wissens gar nicht erst entstehen lassen und den Gettierfall - ohne Kenntnis des Sprechers - ausgeschlossen. Analog in Chisholms Beispiel: Schon eine Ersetzung der Äußerung „Es steht ein Schaf auf der Wiese“ durch „Dies da ist ein Schaf" hätte den Gettierfall vermieden.

Gettierfälle zumindest eines bestimmten Typs scheinen sich eliminieren zu lassen, wenn es gelingt, die Überzeugung auf eine solche Weise zu formulieren, dass die versteckte Fehlannahme auf der sprachlichen Oberfläche erscheint, so dass das, was an ihren Rechtfertigungsgründen, Implikationen oder Präsuppositionen irrig ist, auf den Wahrheitswert des Gesagten durchschlägt - kurz: wenn es gelingt, die wahre in eine falsche Überzeugung zu überführen. Dies wird durch eine demonstrative Bezeichnung des Redegegenstands bewerkstelligt, und zwar auf eine Art, die doxastisch äquivalent mit der ursprünglichen Formulierung ist. Denn dass „dieser Mann da“, Jones, den Job bekommt, hat Smith ohnehin geglaubt.

Freilich kann der Sprecher nicht wissen, welches Situationsmerkmal er jeweils demonstrativ bezeichnen muss, um das Gesagte der Falsifikation auszusetzen, da sich die Komplikation ja in seinem blinden Fleck befindet. Der sprachliche Kniff ist weder zur Verbesserung der epistemischen Lage des Sprechers noch zur Verbesserung der Wissensdefinition geeignet.

(iii) Die skizzierte Behandlung des Gettierproblems macht eine Reihe von erkenntnistheoretischen, metaphysischen und begrifflichen Voraussetzungen, für die kaum argumentiert wird, die aber verschiedene (Auf)Lösungen des Gettierproblems ausschließen, so die infallibilistische und die skeptische.

Das ist richtig, aber ist es ein Einwand? Eine Analyse des Wissensbegriffs, eine Lösung des Gettierproblems und schon eine Charakterisierung von Gettierfällen müssen auch in die philosophische Landschaft passen. Weil der 
Wissensbegriff eine so zentrale Stellung in unserem Begriffssystem einnimmt, steht sein angemessenes Verständnis unter strengen Restriktionen. Man kann nicht isoliert das Gettierproblem lösen oder auch nur erhellend kommentieren wollen, ohne sich auf eine Reihe von Annahmen über die menschliche Fehlbarkeit, die Natur der Wahrheit, den Begriff der Rechtfertigung und einiges andere zu verpflichten. Das Habitat des Wissensbegriffs ist schon dicht besiedelt, und da der Begriff verträglich mit seinen Begriffsnachbarn zusammenleben soll, ist sein Spielraum gering. ${ }^{48}$

Im Einzelnen: Die infallibilistische Reaktion auf das Gettierproblem, derzufolge in Gettierfällen keine gerechtfertigte Überzeugung vorliegt, weil Rechtfertigungen die Wahrheit des Geglaubten garantieren müssen, passt nicht in die philosophische Landschaft, weil es keine guten Gründe für die Annahme gibt, dass Menschen über wahrheitsgarantierende Rechtfertigungsverfahren verfügen. Der Begriff einer garantiert wahren gerechtfertigten Meinung entspricht auch nicht dem gewöhnlichen Begriff des Wissens.

Wer der Auffassung ist, dass man niemals darin gerechtfertigt sein kann, etwas Falsches zu glauben, muss überdies den Rechtfertigungsbegriff zurechtbiegen. Er muss zahllose Fälle, in denen Personen einander Gerechtfertigtsein zuschreiben, als Fehlzuschreibungen ansehen.

Die Kombination eines infallibilistischen Wissensbegriffs mit der fallibilistischen Annahme, dass Menschen über keine wahrheitsgarantierenden Rechtfertigungsverfahren verfügen, führt zur Skepsis: zur Auffassung, dass Menschen kein Wissen besitzen, weil ihre Rechtfertigungen niemals gut genug sind. Ein Skeptiker muss und kann das Gettierproblem nicht lösen, weil er von vornherein die Gettier-Intuition bestreitet. ${ }^{49}$

Auch Safety-Theorien und Antizufallstheorien müssen einen modus vivendi mit dem Fallibilismus finden. Sie laufen Gefahr, unter Realbedingungen die skeptische Auffassung nach sich zu ziehen, dass Menchen kaum etwas oder nichts wissen.

(iv) Wenn man sowohl den infallibilistischen Wissensbegriff als auch die Skepsis ablehnt, bleibt die Auffassung übrig, dass Menschen Wissen besitzen, ohne über wahrheitsgarantierende Rechtfertigungsverfahren zu verfügen. Das würde bedeuten, dass Wissen mit Glück verträglich ist. Beruht aber die Gettier-Intuition nicht gerade auf der Annahme, dass Wissen nicht mit Glück verträglich ist?

\footnotetext{
${ }^{48}$ Diese Bemerkungen sind dem Projekt der „konnektiven Analyse“ (Strawson) philosophischer Grundbegriffe verpflichtet.

${ }^{49}$ Diese Darstellung der Zusammenhänge zwischen infallibilistischem Wissensbegriff, Fallibilismus und Skepsis ist unoriginell; vgl. z. B. Bernecker $(2013,36)$.
} 
In der jüngeren Phase der Gettierdebatte ist intensiv darüber diskutiert worden, welche Art von Zufall in Gettierfällen involviert ist und welche epistemische Rolle er dort spielt. Im Zuge einer genaueren Bestimmung der Zufallskomponente in Gettierfällen sind etliche Teilnehmer der Debatte (z. B. Pritchard, Hetherington und Baumann) zu der Auffassung gelangt, dass nicht jede Art und jedes Ausmaß an Glück mit Wissen unverträglich ist. ${ }^{50}$

In Gettierfällen ist eine charakteristische Kombination von Glück und Pech am Werk, die sich mit Zagzebski $(1994,69)$ so beschreiben lässt, dass ein unglücklicher Zufall ohne Wissen des Subjekts die Rechtfertigung untergräbt, während die resultierende Überzeugung aufgrund eines glücklichen Zufalls gleichwohl wahr ist. Gettierfälle sind also „Fälle von epistemischem Glück im Unglück“ (Brendel 2013, 51).

Betrachten wir nun diejenigen nichtinferentiellen Gettierfälle, in denen das Subjekt in Unkenntnis der Anwesenheit perfekter Attrappen, seien es solche von Scheunen oder von Schafen, eine wahrnehmungsinduzierte wahre Überzeugung erwirbt. Wenn die Anwesenheit der Gettierkomplikation epistemisches Pech ist, das Wissen ausschließt, warum soll man dann nicht die Abwesenheit einer Gettierkomplikation einen Fall von epistemischem Glück nennen? Dass das Subjekt weder von seinem Glück noch von seinem Pech etwas ahnt, hat ja in beiden Fällen denselben Grund, nämlich die subjektive Ununterscheidbarkeit von Attrappen und Scheunen. Wenn man im glücklichen Fall Wissen zuschreibt, dann scheint Wissen jedenfalls mit einer Art von Glück vereinbar zu sein, nämlich mit der glücklichen Abwesenheit einer Gettierkomplikation.

Der Skeptiker sieht freilich schon in der subjektiven Ununterscheidbarkeit von veridischer und nichtveridischer Wahrnehmung einen mit Wissen unvereinbaren Rechtfertigungsmangel. Darin sollte man ihm nicht folgen. Dass die subjektive Ununterscheidbarkeit nicht schon als rechtfertigungs- und wissensunterminierend gilt, illustriert die bekannte Anekdote über Voltaire: Als Voltaire auf einer Kutschenfahrt mit einem Freund an einer Weide vorbeikommt, bemerkt der Freund: „Die Schafe sind frisch geschoren!“. Darauf Voltaire: „Zumindest von einer Seite.” - Die Auffassung, dass man bei der Betrachtung einer Schafherde nicht wisse, ob die Schafe einseitig oder komplett geschoren sind, weil man sie schließlich nur von einer Seite sieht, erscheint als eigenwillige und unpassende Erhöhung von Rechtfertigungsstandards. Dass diese Erhöhung trotz der postulierten subjektiven Ununterscheidbarkeit beider Fälle als unpassend empfunden wird, hat einen Grund, der auch für die Asymmetrie zwischen dem epistemischen Pech der Gettierkomplikation und dem Glück ihres Ausbleibens wichtig ist. Bisher habe ich nur

${ }^{50}$ Zum Überblick vgl. z. B. Brendel (2013) und Baumann (2013). 
die Symmetrie betont, dass Gettierfälle Fälle von unglücklichem Nichtwissen sind, die ohne die Anwesenheit der Gettierkomplikation Fälle von glücklichem Wissen gewesen wären. Das Gemeinsame beider Fälle ist die Abhängigkeit meines Wissensstatus von einem kontingenten, von mir nicht kontrollierten und mir nicht zurechenbaren Faktor.

Die Asymmetrie besteht darin, dass man viel Pech braucht, um einem Gettierfall zum Opfer zu fallen, aber nur wenig Glück, um ihm zu entgehen. Damit es diese Asymmetrie geben kann, muss noch ein anderer Begriff von Zufall im Spiel sein. Plausiblerweise ist es der probabilistische: Gettierkomplikationen sind, wie perfekte Attrappen, in unserer Welt statistisch selten. ${ }^{51}$ Man kann deshalb zugeben, dass beim Wissen etwas Glück im Spiel ist, ohne zugeben zu müssen, dass in Gettierfällen Wissen vorliegt, denn dort ist ein seltenes Ausmaß an Pech im Spiel, genauer: die beschriebene Kombination von epistemischem Glück im Unglück.

Natürlich ist das eine Common sense-Einschätzung. Ein cartesianischer Skeptiker wird die Asymmetrie mit dem Argument leugnen, dass man schließlich nicht wissen könne, dass es nur wenige perfekte Attrappen gibt. In diesem skeptischen Einwand ist aber der (zu) anspruchsvolle, infallibilistische Wissensbegriff schon vorausgesetzt. ${ }^{52}$

\section{LITERATUR}

Austin, John L. (1956): „A Plea for Excuses”, zitiert nach ders.: Philosophical Papers, Oxford ${ }^{3} 1979,175-204$.

Baumann, Peter (2013): „Gettier, Wissen, Zufall”, in diesem Band, 9-27.

Bealer, George (1996): „A Priori Knowledge and the Scope of Philosophy”, Philosophical Studies 81, 121-142.

- (1998): „Intuition and the Autonomy of Philosophy”, in: DePaul, Michael R.; Ramsey, William (Hg.): Rethinking Intuition: The Psychology of Intuition and Its Role in Philosophical Inquiry, Lanham, MD, 201-239.

Bernecker, Sven (2013): „Warum das Gettier-Problem kein Scheinproblem ist", in diesem Band, 29-48.

Brendel, Elke (2013): „Gettier und die Frage nach der Vereinbarkeit von Wissen und Zufall”, in diesem Band, 49-68.

\footnotetext{
${ }^{51}$ So argumentiert auch Baumann (2013, 21-24). Hinsichtlich des Ausmaßes des wissensverträglichen Zufalls argumentiert er kontextualistisch: Die Festlegung eines bestimmten Schwellenwertes wäre arbiträr; angemessen erscheint eine Relativierung auf Kontextparameter (vgl. ebd.).

${ }^{52}$ Ich danke Sven Bernecker, Elke Brendel, Alexander Dinges und Nora Kreft für hilfreiche Diskussionen.
} 
Buckwalter, Wesley and Stephen Stich (2011): „Gender and the Philosophy Club", The Philosophers' Magazine 52, 60-65.

Cappelen, Herman (2012): Philosophy Without Intuitions, Oxford.

Chalmers, David and Frank Jackson (2001): „Conceptual Analysis and Reductive Explanation”, Philosophical Review 110, 315-360.

Chisholm, Roderick M. (1989): Theory of Knowledge [1966], Englewood, NJ, 3. Aufl.

Devitt, Michael (2006): Ignorance of Language, Oxford.

- (2013): „Linguistic intuitions are not ,the voice of competence“”, in: Haug, Matthew (Hg.): Philosophical Methodology: The Armchair or the Laboratory?, London, 269-293.

Gettier, Edmund L. (1963): „Is Justified True Belief Knowledge?“, Analysis $23,121-123$.

Goldman, Alvin I. (1976): „Discrimination and Perceptual Knowledge”, Journal of Philosophy 73, 771-791.

- (2007): „Philosophical Intuitions: Their Target, Their Source, and Their Epistemic Status", Grazer Philosophische Studien 74, 1-25.

Grice, H. Paul (1958): „Postwar Oxford Philosophy”, zitiert nach ders., Studies in the Way of Words, Cambridge, MA/London 1989, 171-180.

Hetherington, Stephen (2001): Good Knowledge, Bad Knowledge: On Two Dogmas of Epistemology, Oxford.

Ichikawa, Jonathan (2009): „Knowing the Intuition and Knowing the Counterfactual“", Philosophical Studies 145, 435-443.

Ingarden, Roman (1968): Vom Erkennen des literarischen Kunstwerks, Tübingen.

Jackson, Frank (1998): From Metaphysics to Ethics. A Defence of Conceptual Analysis, Oxford.

Katz, Leo (2011): Why the Law Is So Perverse, Chicago.

Keil, Geert (2005): „Über die deskriptive Unerschöpflichkeit der Einzeldinge“, in: Keil, Geert; Tietz, Udo (Hg.): Phänomenologie und Sprachanalyse, Paderborn, 83-125.

- (2007): „What Do Deviant Causal Chains Deviate From?”, in: Lumer, Christoph; Nannini, Sandro (Hg.): Intentionality, Deliberation and $\mathrm{Au}-$ tonomy, Aldershot, 69-90.

Martens, David B. (2011): „A Late Medieval Dispute about the Conditions for Knowledge“, Philosophical Papers 40.3, 421-438.

Nagel, Jennifer (2007): „Epistemic Intuitions“, Philosophy Compass 2.6 (2007), 792-819.

- (2012): „Intuitions and Experiments: A Defense of the Case Method in Epistemology", Philosophy and Phenomenological Research 85.3, 495527. 
Nagel, Jennifer, Valerie San Juan and Raymond A. Mar (2013): „Lay Denial of Knowledge for Justified True Beliefs", Cognition, zuerst online: http://www.sciencedirect.com/science/article/pii/S001002771300036X.

Pardo, Michael S. (2010): „The Gettier Problem and Legal Proof”, Legal Theory 16, 37-57.

Platon (Theaitet): Theaitetos, übers. v. F. Schleiermacher, Sämtliche Werke Bd. 4, Hamburg 1958.

Pritchard, Duncan (2005): Epistemic Luck, Oxford.

Quine, W. V. O. (2000): „Quine's Responses“, in: Orenstein, Alex; Kotatko, Petr (Hg.): Knowledge, Language and Logic. Questions for Quine, Dordrecht/Boston/London, 407-430.

Russell, Bertrand (1948): Human Knowledge. Its Scope and Limits, London.

Sosa, Ernest (1991): Knowledge in Perspective, Cambridge.

- (1998): „Minimal Intuition”, in: DePaul, Michael R.; Ramsey, William (Hg.): Rethinking Intuition: The Psychology of Intuition and Its Role in Philosophical Inquiry, Lanham, MD, 257-269.

- (2007): „Intuitions: Their Nature and Epistemic Efficacy”, Grazer Philosophische Studien 74, 51-67.

Stoltz, Jonathan (2007): „Gettier and Factivity in Indo-Tibetan Epistemology”, Philosophical Quarterly 57, 394-415.

Tolksdorf, Stefan (2013): ,There really is no such thing as a ,Gettier challenge“", unveröffentlichtes Manuskript.

Tugendhat, Ernst (2007): Anthropologie statt Metaphysik, München.

Urmson, James O. (1969): „A Symposium on Austin's Method”, in: Fann, K. T. (Hg.): Symposium on J. L. Austin, London, 76-86.

Weatherson, Brian (2003): „What Good are Counterexamples?”, Philosophical Studies 115, 1-31.

Weinberg, Jonathan M., Shaun Nichols and Stephen Stich (2001), „Normativity and Epistemic Intuitions", Philosophical Topics 29, 429-460.

Williamson, Timothy (2000): Knowledge and its Limits, Oxford.

- (2007): The Philosophy of Philosophy, Oxford.

- (2009): „Probability and Danger“, The Amherst Lecture in Philosophy 4, 1-35, http://www.amherstlecture.org/williamson2009/.

Wittgenstein, Ludwig (PU): Philosophische Untersuchungen [1953], Frankfurt am Main 1960.

Zagzebski, Linda (1994): „The Inescapability of Gettier Problems”, The Philosophical Quarterly 44, 56-73. 